\title{
Pacific
}

Journal of

Mathematics

FACTORIZATION PROBLEMS IN THE INVERTIBLE GROUP OF A HOMOGENEOUS $C^{*}$-ALGEBRA

N. Christopher PHILLIPS 


\section{FACTORIZATION PROBLEMS IN THE INVERTIBLE GROUP OF A HOMOGENEOUS C*-ALGEBRA}

\section{N. Christopher Phillips}

Let $X$ be a compact metric space of dimension $d$. In previous work, we have shown that for all sufficiently large $n$, every element of the identity component $U_{0}\left(C(X) \otimes M_{n}\right)$ of the unitary group $U\left(C(X) \otimes M_{n}\right)$ is a product of at most 4 exponentials of skewadjoint elements. On the other hand, if $X$ is a manifold then some elements of $U_{0}\left(C(X) \otimes M_{n}\right)$ require at least about $d / n^{2}$ exponentials. Similar qualitative behavior (with different bounds: 5 and $\left.d /\left(2 n^{2}\right)\right)$ holds for the problem of factoring elements of the identity component $\operatorname{inv}_{0}\left(C(X) \otimes M_{n}\right)$ of the invertible group as products of exponentials of arbitrary elements of the algebra. In this paper, we identify the sets of finite products of 10 other types of elements of $\operatorname{inv}_{0}\left(C(X) \otimes M_{n}\right)$, and we show that the minimum lengths of factorizations have the same qualitative behavior as the two exponential factorization problems above (after a suitable minor modification in 3 of the 10 cases). We obtain upper bounds for large $n$ that range from 5 to 22 , and lower bounds approximately of the form $r d / n^{2}$ with $r$ ranging from $1 / 16$ to 2 . The classes of elements we consider all make sense in general unital $\mathrm{C}^{*}$ algebras. They are: unipotents, positive invertibles, selfadjoint invertibles, symmetries, ${ }^{*}$-symmetries, commutators of elements of $\operatorname{inv}_{0}(A)$ and $U_{0}(A)$, accretive elements, accretive unitaries, and positive-stable elements (real part of spectrum positive). The last three classes are the ones requiring the slight modification; without it, lengths of factorization behave like exponential length rather than exponential rank.

\section{Introduction.}

If $A$ is a unital $\mathrm{C}^{*}$-algebra, then the $\mathrm{C}^{*}$ exponential rank of $A$, denoted $\operatorname{cer}(A)$, is the smallest $n \in\{1,1+\varepsilon, 2,2+\varepsilon, \ldots, \infty\}$ such that every element of the identity componcnt of the unitary group of $A$ is a product of at most $n$ exponentials of skewadjoint elements. (We say $u$ is a product of $k+\varepsilon$ exponentials if it is a limit of products of $k$ exponentials.) In [13] it is proved that for $n \geq 2$ and $X$ a compact manifold, $\operatorname{cer}\left(C(X) \otimes M_{n}\right)$ is at least as large as about $\operatorname{dim}(X) / n^{2}$, but on the other hand is bounded above 
by a finite function of $n$ and $\operatorname{dim}(X)$ which is at most 4 for $n$ sufficiently large (depending on $d$ ).

In this paper, we consider the problem of factoring suitable invertible elements of $C(X) \otimes M_{n}$ as products of a number of other kinds of factors, such as positive invertible elements, unipotent elements, symmetries, and commutators of homotopically trivial invertibles and unitaries. Including exponential rank and several other problems that have been studied before, we consider a total of 12 factorization problems. (A detailed list is given at the beginning of Section 1.) We prove in this paper that 9 of them have the same qualitative behavior on $C(X) \otimes M_{n}$ as described above for $\mathrm{C}^{*}$ exponential rank (although with different constants). The remaining 3 problems behave like $\mathrm{C}^{*}$ exponential length [24], but, after a suitable small modification, they too behave as above. Including the already known cases for completeness, we thus give 12 theorems which say essentially the same thing about different factorization problems in $C(X) \otimes M_{n}$.

We find it striking that 9 different factorization problems, and slight modifications of 3 others, all have the same qualitative behavior on algebras of the form $C(X) \otimes M_{n}$. In particular, the same topological obstruction seems to prevent short factorizations when $\operatorname{dim}(X)>>n \geq 2$ (even when $X$ is contractible), and then seems to disappear for large $n$.

In Section 1, we describe 13 different factorization problems. (We only get 12 theorems because for $C(X) \otimes M_{n}$, although not in general $\mathrm{C}^{*}$-algebras, two of the problems turn out to be identical.) We introduce notation, prove several general lemmas, and discuss known results on the factorization problems for $M_{n}$ and $L(H)$. The remaining three sections contain the proofs of our theorems; the arrangement is described near the end of Section 1.

Unless otherwise specified, we consider only compact metric spaces $X$; in some of our problems, this saves some technicalities involving dimensions of compact spaces that are not second countable. If $a: X \rightarrow M_{n}$ is a function, then $\operatorname{det}(a)$ is the function $x \mapsto \operatorname{det}(a(x))$. We let $S U_{n}$ and $S L_{n}$ denote the unitaries and invertibles in $M_{n}$ with determinant 1 . If $Z$ is any metric space (for example, $S U_{n}$ ), then $C(X, Z)$ denotes the space of continuous functions from $X$ to $Z$ with the topology of uniform convergence.

I would like to thank Terrance Quinn for valuable e-mail correspondence, and Ian Putnam for calling my attention to Quinn's work on factorizations into positive elements. It was questions about such factorizations that led to this paper. 


\section{Generalities on Factorization.}

Let $A$ be a unital $\mathrm{C}^{*}$-algebra, let $U(A)$ and $\operatorname{inv}(A)$ be its unitary and invertible groups, and let $U_{0}(A)$ and $\operatorname{inv}_{0}(A)$ be their identity components. In this paper, we consider the lengths of factorizations of elements of $\operatorname{inv}_{0}(A)$, when the factorization is possible at all, into selfadjoint invertible elements, positive invertible elements, commutators of elements of $U_{0}(A)$ or $\operatorname{inv}_{0}(A)$, exponentials of skewadjoint or arbitrary elements of $A$, and the classes of operators given in the following definition. (The terminology in the definition specializes for $M_{n}$, except as noted, to that used in the survey article [29] on factorization problems in $M_{n}$.)

1.1 Definition. Let $A$ be a unital $\mathrm{C}^{*}$-algebra. Then an element $a \in A$ is called:

(1) a symmetry ("involution" in [29]) if $a^{2}=1$;

(2) a *-symmetry ("symmetry" in [29]) if $a$ is unitary and $a^{2}=1$;

(3) unipotent if $a-1$ is nilpotent;

(4) quasiunipotent (not in [29]) if $a-1$ is quasinilpotent;

(5) positive-stable if $\operatorname{sp}(a) \subset\{\lambda \in \mathbb{C}: \operatorname{Re}(\lambda)>0\}$;

(6) accretive if $\left(a+a^{*}\right) / 2$ is positive and invertible;

(7) unitary accretive (not in [29]) if a is accretive and unitary.

We point out that a positive-stable (written without the hyphen in [29]) element is not necessarily positive. Our definition of accretive agrees with that in [29] but conflicts with terminology used elsewhere. (See Section 4.) To clarify the significance of accretive and positive-stable elements, we prove in Section 4 that an accretive element is positive-stable (and hence invertible), and that $a \in A$ is accretive if and only if $\operatorname{Re}(\varphi(a))>0$ for every state $\varphi$.

Including the ones mentioned before the definition, there are altogether 13 classes. Somewhat over half of them are discussed in [29] for $M_{n}$ and (sometimes) for $L(H)$. Unlike [29], we insist that positive and selfadjoint elements be invertible, and, rather than using the most obvious generalization of [29], we use commutators of elements of $\operatorname{inv}_{0}(A)$. Of the remaining classes, products of exponentials are trivial in $M_{n}$. Unitary commutators seem to have been overlooked in [29]. Quasiunipotent elements seem to be a more natural class than unipotent elements in a $\mathrm{C}^{*}$-algebra, but in $M_{n}$ (the primary focus of [29]) and in $C(X) \otimes M_{n}$ (the primary focus here), both classes are the same. Finally, we consider accretive unitaries because of their close connection with exponential length. (See Section 4.)

The paper [29] discusses a large number of other factorization problems. 
Some, such as products of symmetric matrices and pseudoinvolutions, do not make sense in abstract $\mathrm{C}^{*}$-algebras without additional structure. Others, such as products of projections, partial isometries, and nilpotent elements, make sense in an abstract $\mathrm{C}^{*}$-algebra but do not take place in $\operatorname{inv}_{0}(A)$, even with simple modifications. The problem of factorization into normal elements, also considered in [29], can be easily modified to take place in $\operatorname{inv}_{0}(A)$, but it then has a trivial solution, given by the polar decomposition.

Only a few factorization problems have been considered in more general $\mathrm{C}^{*}$-algebras. The two exponential factorizations were formally introduced in $[\mathbf{1 1}]$ and $[\mathbf{1 8}]$, and there are now quite a few results; see the survey article [16]. Commutators have also been considered by a number of authors; references are also given in [16]. Factorizations into positive elements have recently been studied by Quinn $[\mathbf{1 9}],[\mathbf{2 0}]$. Unipotent triangular matrices are used in [5] in a purely algebraic setting, which is nevertheless very useful to us, but we do not include this class with the 13 classes above because it is not intrinsic.

1.2 Definition. Let $\mathcal{C}$ be one of the 13 classes of invertible elements of a $\mathrm{C}^{*}$-algebra mentioned before or in Definition 1.1. Let $A$ be a unital $\mathrm{C}^{*}$-algebra. We define the following sets and numbers:

(1) $P_{\mathcal{C}}(A)$ is the set of all finite products of elements of $A$ of the class $\mathcal{C}$.

(2) $\bar{P}_{\mathcal{C}}(A)$ is the closure of $P_{\mathcal{C}}(A)$ in $\operatorname{inv}(A)$.

(3) $\operatorname{rk}_{\mathcal{C}}(A)$ is the smallest number $n$ such that every element of $P_{\mathcal{C}}(A)$ is a product of at most $n$ elements in the class $\mathcal{C}$; it is $\infty$ if no such $n$ exists. We call it the $\mathcal{C}$-rank of $A$.

(4) $\overline{\operatorname{rk}}_{\mathcal{C}}(A)$ is the smallest integer $n$ such that products of $n$ elements of the class $\mathcal{C}$ are dense in $\bar{P}_{\mathcal{C}}(A)$; it is $\infty$ if no such $n$ exists. We call it the $\overline{\mathcal{C}}$-rank of $A$.

Unlike our definition of exponential rank [11], [18], we do not allow the values $n+\varepsilon$ here. We exclude them partly for simplicity and partly because for some of the classes we consider, a limit of products of $n$ members of the class seems unlikely to be a product of $n+1$ members of the class. For example, if $\mathcal{C}$ is the class of positive invertible elements, then Theorem 3 of [10] shows that $\overline{\mathrm{rk}}_{\mathcal{C}}(L(H)) \leq 5$. However, until very recently the best known upper bound for $\operatorname{rk}_{\mathcal{C}}(L(H))$ was 17. (See [30]. This has just been improved to 7 in $[\mathbf{1 7}]$. But it is still not clear whether $\operatorname{rk}_{\mathcal{C}}(L(H))=\overline{\mathrm{rk}}_{\mathcal{C}}(L(H))$.)

If $\mathcal{C}$ is the class of exponentials of skewadjoint elements, then the exponential rank can be recovered as follows:

$$
\begin{array}{lll}
\operatorname{cer}(A)=n & \text { if and only if } & \operatorname{rk}_{\mathcal{C}}(A)=\overline{\operatorname{rk}}_{\mathcal{C}}(A)=n . \\
\operatorname{cer}(A)=n+\varepsilon & \text { if and only if } & \operatorname{rk}_{\mathcal{C}}(A)=n+1 \text { and } \overline{\operatorname{rk}}_{\mathcal{C}}(A)=n .
\end{array}
$$


The same thing can be done whenever the elements of a general class $\mathcal{C}$ include a neighborhood of 1 in $P_{\mathcal{C}}(A)$.

1.3 Proposition. Let $\mathcal{C}$ be any of the 13 classes mentiond in or before Definition 1.1. Then $P_{\mathcal{C}}(A) \subset \bar{P}_{\mathcal{C}}(A) \subset \operatorname{inv}_{0}(A)$ for every unital $\mathrm{C}^{*}$-algebra $A$.

Proof. We show that all the elements of class $\mathcal{C}$ are in $\operatorname{inv}_{0}(A)$. The proposition will then follow from the fact that $\operatorname{inv}_{0}(A)$ is a closed subgroup of $\operatorname{inv}(A)$.

The commutators are in $\operatorname{inv}_{0}(A)$ by definition. It is a standard fact that the exponentials are in $\operatorname{inv}_{0}(A)$. All the other classes satisfy conditions ensuring that the spectrums of their elements do not separate 0 from $\infty$; thus, their elements are all exponentials. (For the classes of accretive elements and accretive unitaries, use Corollary 4.3 below to see this.)

If $H$ is a separable infinite dimensional Hilbert space, then in almost all cases $P_{\mathcal{C}}(L(H))$ is known to be either $U(L(H))$ or inv $(L(H))$, depending only on whether all the elements of the class $\mathcal{C}$ are unitaries or not. (See [29]. For the classes of positive-stable elements and accretive elements, this follows by comparison with the class of positive invertible elements. For the class of accretive unitaries, see Section 4.) In particular, $\overline{P_{\mathcal{C}}}(L(H))=P_{\mathcal{C}} L(H)$ ). Furthermore, in these cases $\operatorname{rk}_{\mathcal{C}}(L(H))<\infty$, with known upper bounds varying from 1 (for exponentials of skewadjoint elements) to 7 (for positive invertible elements); again, see [29] and Section 10 of [16].

For $A=M_{n}$, things are a little more complicated. It turns out that $P_{\mathcal{C}}(A)$ is always closed in $\operatorname{inv}\left(M_{n}\right)$, and always has the form $\{a \in G: \operatorname{det}(a) \in H\}$, where $G$ is either $U\left(M_{n}\right)$ or $\operatorname{inv}\left(M_{n}\right)$, and $H$ is a closed subgroup of the multiplicative group $\mathbb{C}-\{0\}$, not depending on $n$. Generally $\operatorname{rk}_{\mathcal{C}}\left(M_{n}\right)$ is bounded above by a small constant independent of $n$ (between 1 and 5), and often this constant is known to be the exact value, except for the trivial case $n=1$.

For commutative $\mathrm{C}^{*}$-algebras these problems are almost all trivial, but an important distinction arises.

1.4 Proposition. Let $X$ be compact Hausdorff (not necessarily metric), and let $\mathcal{C}$ be one of the 13 classes considered in and before Definition 1.1.

(1) If $X$ is not totally disconnected, and $\mathcal{C}$ is the class of positive-stable, accretive, or unitary accretive elements, then $\operatorname{rk}_{\mathcal{C}}(C(X))=\overline{\mathrm{rk}}_{\mathcal{C}}(C(X))=$ $\infty$.

(2) If $\mathcal{C}$ is any of the remaining 10 classes, then $\operatorname{rk}_{\mathcal{C}}(C(X))=\overline{\operatorname{rk}}_{\mathcal{C}}(C(X))=$ 1 for any $X$. 
Part (2) is obvious and its proof is omitted. Part (1) will be proved in Section 4, where results for totally disconnected $X$ will also be given.

We now turn to the case $C(X) \otimes M_{n}$, with $n \geq 2$. Some more notation is required to state our results:

1.5 Definition. For each of our 13 classes $\mathcal{C}$, we define

$$
N_{\mathcal{C}}(n, d)=\sup _{\operatorname{dim}(X) \leq d} \operatorname{rk}_{\mathcal{C}}\left(C(X) \otimes M_{n}\right)
$$

where $X$ runs through all compact metric spaces whose covering dimension [6] $\operatorname{dim}(X)$ is at most $d$.

We note that, for compact metric spaces, all three of the usual dimensions agree. See Theorem 1.7.7 of [6].

For each of the 10 classes of Proposition 1.4 (2), we will prove a result of the following form:

(1) For any compact metric space $X$, we have

$$
P_{\mathcal{C}}\left(C(X) \otimes M_{n}\right)=\bar{P}_{\mathcal{C}}\left(C(X) \otimes M_{n}\right)
$$

and both are equal to

$$
\left\{a \in \operatorname{inv}_{0}\left(C(X) \otimes M_{n}\right): a(x) \in P_{\mathcal{C}}\left(M_{n}\right) \text { for all } x \in X\right\} .
$$

(2) $\quad N_{\mathcal{C}}(n, d)<\infty$ for all $n \geq 1$ and $0 \leq d<\infty$.

(3) For each fixed $d<\infty$, we have $\lim _{n \rightarrow \infty} \sup N_{\mathcal{C}}(n, d) \leq N_{\mathcal{C}}$, for some explicitly given finite number $N_{\mathcal{C}}$ (betwen 4 and 22, depending on $\mathcal{C}$ ).

(4) If $X$ is a compact manifold with boundary, and $n \geq 2$, then

$$
\overline{\mathrm{rk}}_{\mathcal{C}}\left(C(X) \otimes M_{n}\right) \geq f_{\mathcal{C}}(n, \operatorname{dim}(X)),
$$

for some explicitly given function $f_{\mathcal{C}}$ with $f_{\mathcal{C}}(n, d) \rightarrow \infty$ linearly as $d \rightarrow \infty$ for each fixed $n$.

Note that $\overline{\mathrm{rk}}_{\mathcal{C}}(A) \leq \mathrm{rk}_{\mathcal{C}}(A)$ for any $\mathcal{C}$ and $A$.

For the remaining three classes, (1) still holds, but $\operatorname{rk}_{\mathcal{C}}\left(C(X) \otimes M_{n}\right)$ is generally infinite. We will, however, still recover analogs of (2), (3), and (4) by restricting to appropriate commutators, or, in this context, elements with determinant 1 .

The proofs of these results (except for several that have already been proved elsewhere) occupy the remaining three sections. The three slightly exceptional classes are treated together in Section 4, where their connection with exponential length [24], [18] is demonstrated. The other classes of nonunitary elements are treated in the next section, where the lower bounds 
are shown to follow immediately from results on Banach exponential rank, while most of the upper bounds can be obtained from a theorem of Dennis and Vaserstein on factorization into unipotent triangular matrices. The remaining unitary cases are treated in Section 3. Again, lower bounds follow easily from exponential rank results, but upper bounds require more work.

The bounds we present, both upper and lower, are the best we can easily obtain given the results already known. We have not seriously attempted to find the best possible bounds in any of the problems we consider. We have also not investigated more general $\mathrm{C}^{*}$-algebras. Thus, we can state three problems (out of many possible):

1.6 Problem. Improve the upper and lower bounds given in this paper for $\operatorname{rk}_{\mathcal{C}}\left(C(X) \otimes M_{n}\right)$ for the various classes $\mathcal{C}$ considered.

1.7 Problem. For those classes $\mathcal{C}$ for which it is not obvious, characterize $P_{\mathcal{C}}(A)$ and $\bar{P}_{\mathcal{C}}(A)$ for an arbitrary unital $\mathrm{C}^{*}$-algebra $A$.

Of course, these two problems are not equally interesting for all classes $\mathcal{C}$.

1.8 Problem. Does there exist a $\mathrm{C}^{*}$-algebra $A$ such that $\operatorname{rk}_{\mathcal{C}}(A)$ is finite for one of the 10 classes in Proposition 1.4(2), but infinite for another one?

\section{Factorization of invertible elements.}

We start this section by stating the factorization theorem for exponentials, in effect proved in [13]. We then prove the factorization theorems for unipotent elements, positive invertible elements, selfadjoint invertible elements, symmetries, and commutators of elements of $\operatorname{inv}_{0}(A)$.

The lower bounds in these results are obtained from the lower bound for exponentials, and the upper bounds are obtained from a factorization theorem for upper and lower triangular unipotent matrices due to Dennis and Vaserstein [5]. (The upper bounds for commutators are already in [5].)

2.1 Theorem. Let $\mathcal{C}$ be the class of exponentials. Then:

(1) $\quad P_{\mathcal{C}}\left(C(X) \otimes M_{n}\right)=\bar{P}_{\mathcal{C}}\left(C(X) \otimes M_{n}\right)=\operatorname{inv}_{0}\left(C(X) \otimes M_{n}\right)$.

(2) $N_{\mathcal{C}}(n, d)<\infty$ for $n \geq 1$ and $0 \leq d<\infty$.

(3) For fixed $d<\infty$, we have $\lim _{n \rightarrow \infty} \sup N_{\mathcal{C}}(n, d) \leq 5$.

(4) If $X$ is a compact manifold with boundary and $n \geq 2$, then

$$
\overline{\mathrm{rk}}_{\mathcal{C}}\left(C(X) \otimes M_{n}\right) \geq \frac{\operatorname{dim}(X)-2}{2\left(n^{2}-1\right)} .
$$


Proof. (1) is well known (see [2, Proposition 3.4.3]), (2) follows from Corollary 3.2 of [13] and Proposition 4.6 of [11], (3) is Theorem 3.4 of [13], and (4) is Theorem 2.3 of [13].

For later use, it is important to note that the example which proves part (4) of this theorem is actually a homotopically trivial element of $C\left(X, S U_{n}\right)$.

2.2 Proposition. Let $X$ be a compact manifold with boundary, and let $n \geq 2$. Let $l$ be the least integer such that $l \geq[\operatorname{dim}(X)-2] /\left[2\left(n^{2}-1\right)\right]$. Then there is a homotopically trivial $a \in C\left(X, S U_{n}\right)$ which is not a limit-of products of fewer than $l$ exponentials of elements in $C(X) \otimes M_{n}$.

Proof. Let $m=2\left(n^{2}-1\right)(l-1)+2$. Then $m \leq \operatorname{dim}(X)$. The proof of Theorem 2.3 of [13] exhibits a contractible compact subset $X_{0}$ of $\mathbb{R}^{m}$, and $u \in C\left(X_{0}, S U_{n}\right)$, such that $u$ is not a limit of products of fewer than $l$ exponentials. On the other hand, $u \in U_{0}\left(C\left(X_{0}\right) \otimes M_{n}\right)$ since $X_{0}$ is contractible. Therefore $u=\exp \left(i h_{1}\right) \exp \left(i h_{2}\right) \ldots \exp \left(i h_{N}\right)$ for some $N$ and selfadjoint $h_{1}, \ldots, h_{N} \in C\left(X_{0}\right) \otimes M_{n}$. The proof of Lemma 2.3 of [11] (see the claim $\left(^{*}\right)$ there) shows that $h_{1}, \ldots, h_{N}$ may be chosen to have values in

$$
L=\left\{a \in M_{n}: a \text { is selfadjoint and } \operatorname{tr}(a) \in 2 \pi \mathbf{Z}\right\} .
$$

Since $X$ is a manifold with $\operatorname{dim}(X) \geq m$, we can identify $X_{0}$ with some homeomorphic subset of $X$. Since $L$ is topologically the disjoint union of vector spaces and $X_{0}$ is connected, the Tietze extension theorem provides $k_{1}, \ldots, k_{N}: X \rightarrow L$ such that $\left.k_{j}\right|_{X_{0}}=h_{j}$. Then $v=\exp \left(i k_{1}\right) \cdots \exp \left(i k_{N}\right) \in$ $C\left(X, S U_{n}\right)$ is homotopically trivial, since $L$ can be retracted onto $\{(2 \pi r / n) \cdot 1: r \in \mathbf{Z}\}$. By restriction to $X_{0}$, we see that $v$ is not a limit of products of fewer than $l$ exponentials.

The following theorem is not of the form we are concentrating on in this paper, because triangularity is not an intrinsic property of elements of $C(X) \otimes M_{n}$. However, it has in effect been proved elsewhere, and parts (1)(3) will be used to prove the corresponding parts of several later theorems. (Part (4) is included merely for completeness.) Note that the factors are triangular (presumably alternating upper and lower triangular), not merely pointwise triangularizable.

2.3 Theorem. Let $\mathcal{C}$ be the class of unipotent triangular matrices, that is, elements of $A \otimes M_{n}$ which are either upper or lower triangular and whose diagonal entries are all 1 . Then:

(1) $\quad P_{\mathcal{C}}\left(C(X) \otimes M_{n}\right)=\bar{P}_{\mathcal{C}}\left(C(X) \otimes M_{n}\right)=\left\{a \in \operatorname{inv}_{0}\left(C(X) \otimes M_{n}\right): \operatorname{det}(a)=\right.$ $1\}$. 
(2) $\quad N_{\mathcal{C}}(n, d)<\infty$ for $n \geq 1$ and $0 \leq d<\infty$.

(3) For fixed $d<\infty$, we have $\lim _{n \rightarrow \infty} \sup N_{\mathcal{C}}(n, d) \leq 6$.

(4) If $X$ is a compact manifold with boundary and $n \geq 2$, then

$$
\overline{\operatorname{rk}}_{\mathcal{C}}\left(C(X) \otimes M_{n}\right) \geq \frac{\operatorname{dim}(X)-2}{2\left(n^{2}-1\right)} .
$$

Proof. We first note that it suffices to prove parts (1) and (2) for elementary matrices (unipotent triangular matrices with at most one nonzero offdiagonal entry), since every elementary matrix is unipotent triangular and every unipotent triangular matrix is a product of at most $(n-1)(n-2) / 2$ elementary matrices.

(1) We observe, by factoring out the determinant in a homotopy, that $\left\{a \in \operatorname{inv}_{0}\left(C(X) \otimes M_{n}\right): \operatorname{det}(a)=1\right\}$ is the set of homotopically trivial elements of $C\left(X, S L_{n}\right)$. Indeed, if $a \in \operatorname{inv}_{0}\left(C(X) \otimes M_{n}\right)$ and $\operatorname{det}(a)=1$, we can choose a homotopy $t \mapsto a_{t}$ from $a$ at $t=0$ to 1 at $t=1$. Let $(t, x) \mapsto \xi_{t}(x)$ be the continuous branch of $\operatorname{det}\left(a_{t}(x)\right)^{-1 / n}$ which is 1 when $t=1$. Then $t \mapsto \xi_{t} a_{t}$ is a homotopy in $C\left(X, S L_{n}\right)$ from $a$ to $\xi_{1} a_{1}$, which is locally constant with values of the form $\lambda \cdot 1$ with $\lambda^{n}=1$. Since $S L_{n}$ is connected, it is easy to connect $\xi_{1} a_{1}$ to 1 in $C\left(X, S L_{n}\right)$.

By Lemma 9 of [28], the set of finite products of elementary matrices contains a neighborhood of 1 in $C\left(X, S L_{n}\right)$. It is clearly a connected subgroup, and it is therefore open. So it is the identity component. Therefore the previous paragraph shows that

$$
P_{\mathcal{C}}\left(C(X) \otimes M_{n}\right)=\left\{a \in \operatorname{inv}_{0}\left(C(X) \otimes M_{n}\right): \operatorname{det}(a)=1\right\} .
$$

Since this subset is closed in $\operatorname{inv}\left(C(X) \otimes M_{n}\right)$, it is also equal to $\bar{P}_{\mathcal{C}}(C(X) \otimes$ $\left.M_{n}\right)$.

(2) This follows from Theorem 4 of [28].

(3) The stable rank $\operatorname{sr}(C(X))$ is the greatest integer not exceeding $1+\operatorname{dim}(X) / 2$, by [27]. Using (2), the result now follows from Theorem 20 of [5].

(4) This follows from Proposition 2.2, since every unipotent triangular matrix is an exponential.

As an immediate corollary, we get the theorem on unipotent factorizations.

2.4 Theorem. Let $\mathcal{C}$ be the class of unipotent elements. Then:

(1) $P_{\mathcal{C}}\left(C(X) \otimes M_{n}\right)=\bar{P}_{\mathcal{C}}\left(C(X) \otimes M_{n}\right)=\left\{a \in \operatorname{inv}_{0}\left(C(x) \otimes M_{n}\right): \operatorname{det}(a)=1\right\}$.

(2) $N_{\mathcal{C}}(n, d)<\infty$ for $n \geq 1$ and $0 \leq d<\infty$.

(3) For fixed $d<\infty$, we have $\lim _{n \rightarrow \infty} \sup N_{\mathcal{C}}(n, d) \leq 6$. 
(4)

If $X$ is a compact manifold with boundary and $n \geq 2$, then

$$
\overline{\mathrm{rk}}_{\mathcal{C}}\left(C(X) \otimes M_{n}\right) \geq \frac{\operatorname{dim}(X)-2}{2\left(n^{2}-1\right)}
$$

Proof. Part(4) follows from Proposition 2.2, one inclusion in (1) is immediate, and all the rest follows from the previous theorem.

The following two lemmas enable us to apply Theorem 2.3 to other factorization problems.

2.5 Lemma. Let $a \in C(X) \otimes M_{n}$ be triangular, with diagonal elements $\alpha_{1}(x), \ldots, \alpha_{n}(x)$ at $x \in X$. Assume that, for each $x \in X$, the numbers $\alpha_{j}(x)$ are all distinct. Then there is $s \in \operatorname{inv}_{0}\left(C(X) \otimes M_{n}\right)$ such that sas ${ }^{-1}=$ $\operatorname{diag}\left(\alpha_{1}, \ldots, \alpha_{n}\right)$.

Proof. The proof is the same for upper and lower triangular matrices; we do only the upper triangular case. The proof is by induction on $n$. The case $n=1$ is trivial, so suppose the result has been proved for $n$, and let $a \in C(X) \otimes M_{n+1}$ be upper triangular, with diagonal elements $\left(\alpha_{1}, \ldots, \alpha_{n+1}\right)$ with distinct values at each $x \in X$. We write

$$
a(x)=\left(\begin{array}{cc}
a_{0}(x) & \xi(x) \\
0 & \alpha_{n+1}(x)
\end{array}\right)
$$

with $a_{0} \in C(X) \otimes M_{n}$ upper triangular, and with $\xi: X \rightarrow \mathbb{C}^{n}$ continuous (thinking of elements of $\mathbb{C}^{n}$ as column vectors). By the induction hypothesis, there is $s_{0} \in \operatorname{inv}_{0}\left(C(X) \otimes M_{n}\right)$ such that $s_{0} a_{0} s_{0}^{-1}=\operatorname{diag}\left(\alpha_{1}, \ldots, \alpha_{n}\right)$. Furthermore, $\alpha_{n+1}(x)$ is not an eigenvalue of $a_{0}(x)$, since the $\alpha_{j}(x)$ are distinct. Therefore we can define

$$
s(x)=\left(\begin{array}{cc}
s_{0}(x) & 0 \\
0 & 1
\end{array}\right)\left(\begin{array}{cc}
1 & -\left(\alpha_{n+1}(x)-a_{0}(x)\right)^{-1} \xi(x) \\
0 & 1
\end{array}\right)
$$

for $x \in X$, and one checks that $\operatorname{sas}^{-1}=\operatorname{diag}\left(\alpha_{1}, \ldots, \alpha_{n+1}\right)$.

2.6 Lemma. Let $a \in C(X) \otimes M_{n}$ be the product of $k \geq 2$ unipotent triangular matrices. Then a is a product of $2 k$ positive invertible elements, and also-a product of $2 k$ symmetries.

Proof. Write $a=a_{1} a_{2} \cdots a_{k}$ with $a_{k}$ triangular and unipotent. Let $c$ be any invertible diagonal matrix. Write

$$
a=\left(a_{1} c\right)\left(c^{-1} a_{2} c^{2}\right)\left(c^{-2} a_{3} c^{3}\right) \cdots\left(c^{-(k-2)} a_{k-1} c^{k-1}\right)\left(c^{-(k-1)} a_{k}\right) .
$$


Let $b_{j}$ be the $j$-th factor in this expression. Then each $b_{j}$ is again triangular, and the diagonal entries of $b_{j}$ are the same as those of $c$ (for $j<k$ ) or those of $c^{-(k-1)}$ (for $j=k$ ). If the diagonal entries of $c(x)$ and $c(x)^{-(k-1)}$ are all distinct for each $x \in X$, then Lemma 2.5 provides invertible elements $s_{j}$ such that $s_{j} b_{j} s_{j}^{-1}=c$ for $j<k$ and $s_{j} b_{j} s_{j}^{-1}=c^{-(k-1)}$ for $j=k$.

To write $a$ as a product of $2 k$ positive invertible elements, we now follow the proof of Theorem 2 of [25]. Take $c=\operatorname{diag}\left(\alpha, \ldots, \alpha_{n}\right)$ with $\left(\alpha, \ldots, \alpha_{n}\right)$ distinct positive real numbers. For $j<k$, we then have $b_{j}=\left(s_{j}^{*} s_{j}\right)^{-1}\left(s_{j}^{*} c s_{j}\right)$, a product of two positive invertible elements. For $j=k$, use $c^{-(k-1)}$ in place of $c$ to get the same thing.

To write $a$ as a product of $2 k$ symmetries, we follow the proof of Theorem 5 of [25]. Suppose first that $n$ is even, so $n=2 m$. Let $\lambda_{1}, \ldots \lambda_{m}$ be complex numbers of the form $\lambda_{j}=\exp \left(2 \pi i \theta_{j}\right)$, with $\theta_{1}, \ldots, \theta_{m}$ real, irrational, and algebraically independent over $\mathbf{Q}$. Take $c=\operatorname{diag}\left(\lambda_{1}, \lambda_{1}^{-1}, \lambda_{2}, \lambda_{2}^{-1}, \ldots, \lambda_{m}, \lambda_{m}^{-1}\right)$. Then $c$ and $c^{-(k-1)}$ have all their diagonal entries distinct. As in [25], the equation

$$
\left(\begin{array}{cc}
\lambda & 0 \\
0 & \lambda^{-1}
\end{array}\right)=\left(\begin{array}{cc}
0 & 1 \\
1 & 0
\end{array}\right)\left(\begin{array}{cc}
0 & \lambda^{-1} \\
\lambda & 0
\end{array}\right)
$$

can be used to show that $c$ and $c^{-(k-1)}$ are each products of two symmetries. So $b_{j}$, being similar to $c$ or $c^{-(k-1)}$, is also a product of two symmetries.

If $n$ is odd, let $n=2 m+1$, choose $\lambda_{1}, \ldots, \lambda_{m}$ as before, and take $c=$ $\operatorname{diag}\left(\lambda_{1}, \lambda_{1}^{-1}, \ldots, \lambda_{m}, \lambda_{m}^{-1}, 1\right)$. Then proceed as before.

2.7 Theorem. Let be the class of positive invertible elements. Then:

(1) $\quad P_{\mathcal{C}}\left(C(X) \otimes M_{n}\right)=\bar{P}_{\mathcal{C}}\left(C(X) \otimes M_{n}\right)=\left\{a \in \operatorname{inv}_{0}\left(C(X) \otimes M_{n}\right): \operatorname{det}(a)>\right.$ $0\}$.

(2) $N_{\mathcal{C}}(n, d)<\infty$ for $n \geq 1$ and $0 \leq d<\infty$.

(3) For fixed $d<\infty$, we have $\lim _{n \rightarrow \infty} \sup N_{\mathcal{C}}(n, d) \leq 12$.

(4) If $X$ is a compact manifold with boundary and $n \geq 2$, then

$$
\overline{\mathrm{rk}}_{\mathcal{C}}\left(C(X) \otimes M_{n}\right) \geq \frac{\operatorname{dim}(X)-2}{n^{2}-1}-1
$$

Proof. (1) Since

$$
\left\{a \in \operatorname{inv}_{0}\left(C(X) \otimes M_{n}\right): \operatorname{det}(a)>0\right\}
$$

is closed in $\operatorname{inv}\left(C(X) \otimes M_{n}\right)$ and obviously contains $P_{\mathcal{C}}\left(C(X) \otimes M_{n}\right)$, it is enough to show that it is contained in $P_{\mathcal{C}}\left(C(X) \otimes M_{n}\right)$. So let $a \in$ $\operatorname{inv}_{0}\left(C(X) \otimes M_{n}\right)$ and suppose $\operatorname{det}(a)>0$. Then

$$
\operatorname{det}(a)^{-1 / n} a \in \operatorname{inv}_{0}\left(C(X) \otimes M_{n}\right)
$$


and has determinant 1. By Theorem 2.3(1) and Lemma 2.6, it is a product of positive invertible elements. Therefore so is

$$
a=\left(\operatorname{det}(a)^{1 / n} \cdot 1\right)\left(\operatorname{det}(a)^{-1 / n} a\right) .
$$

(2) This follows from the corresponding part of Theorem 2.3 by using Lemma 2.6 just as above.

(3) Fix $d<\infty$. Combine Lemma 2.6 and Theorem 2.3(3) as in the argument for part (1) to obtain the following statement: For all sufficiently large $n$, all $X$ with $\operatorname{dim}(X)=d$, and all $a \in \operatorname{inv}_{0}\left(C(X) \otimes M_{n}\right)$, there are 12 positive invertible elements $a_{1}, \ldots, a_{12} \in C(X) \otimes M_{n}$ such that $a=\left(\operatorname{det}(a)^{1 / n} \cdot 1\right) a_{1} a_{2} \cdots a_{12}$. Now $\operatorname{det}(a)^{1 / n} a_{1}$ is again a positive invertible element. Thus $a$ is a product of 12 positive invertible elements.

(4) Theorem 2.8 of [29] implies that a product of two positive invertible elements of $M_{n}$ is similar to a positive invertible element of $M_{n}$, and so has spectrum in the right half plane. It follows that the product of two positive invertible elements of $C(X) \otimes M_{n}$ is an exponential. Now use Proposition 2.2 .

2.8 Theorem. Let $\mathcal{C}$ be the class of selfadjoint invertible elements. Then:

(1) $P_{C}\left(C(X) \otimes M_{n}\right)=\bar{P}_{C}\left(C(X) \otimes M_{n}\right)=\left\{a \in \operatorname{inv}_{0}\left(C(X) \otimes M_{n}\right)\right.$ : $\operatorname{det}(a)$ is real\}.

(2) $\quad N_{\mathcal{C}}(n, d)<\infty$ for $n \geq 1$ and $0 \leq d<\infty$.

(3) For fixed $d<\infty$, we have $\lim _{n \rightarrow \infty} \sup N_{\mathcal{C}}(n, d) \leq 13$.

(4) If $X$ is a compact manifold with boundary and $n \geq 2$, then

$$
\overline{\mathrm{rk}}_{C}\left(C(X) \otimes M_{n}\right) \geq \frac{\operatorname{dim}(X)-2}{2\left(n^{2}-1\right)}
$$

Proof. Parts (1), (2), and (3) follow from the corresponding parts of Theorem 2.7 by multiplying by the selfadjoint invertible element

$$
w(x)= \begin{cases}1 & \operatorname{det}(a(x))>0 \\ \operatorname{diag}(-1,1, \ldots, 1) & \operatorname{det}(a(x))<0 .\end{cases}
$$

Part (4) follows from Proposition 2.2, since every selfadjoint invertible element is an exponential.

2.9 Theorem. Let $\mathcal{C}$ be the class of symmetries (called involutions in [25] and [29]). Then:

(1) $P_{\mathcal{C}}\left(C(X) \otimes M_{n}\right)=\bar{P}_{\mathcal{C}}\left(C(X) \otimes M_{n}\right)=\left\{a \in \operatorname{inv}_{0}\left(C(X) \otimes M_{n}\right): \operatorname{det}(a)=\right.$ $\{ \pm 1\}$ for all $x\}$. 
(2) $\quad N_{C}(n, d)<\infty$ for $n \geq 1$ and $0 \leq d<\infty$.

(3) For fixed $d<\infty$, we have $\lim _{n \rightarrow \infty} \sup N_{\mathcal{C}}(n, d) \leq 13$.

(4) If $X$ is a compact manifold with boundary and $n \geq 2$, then

$$
\overline{\mathrm{rk}}_{\mathcal{C}}\left(C(X) \otimes M_{n}\right) \geq \frac{\operatorname{dim}(X)-2}{2\left(n^{2}-1\right)} .
$$

Proof. Parts (1), (2), and (3) are proved in the same way as in the proof of Theorems 2.7 and 2.8. (Note that the element $w$ from the proof of Theorem 2.8 is a symmetry. Also, one uses the part of Lemma 2.6 that refers to symmetries.)

Part (4) follows from Proposition 2.2, since every symmetry is an exponential.

2.10 Theorem. Let $\mathcal{C}$ be the class of multiplicative commutators of elements of $\operatorname{inv}_{0}(A)$. Then:

(1) $P c\left(C(X) \otimes M_{n}\right)=\bar{P}_{\mathcal{C}}\left(C(X) \otimes M_{n}\right)=\left\{a \in \operatorname{inv}_{0}\left(C(X) \otimes M_{n}\right): \operatorname{det}(a)=\right.$ $1\}$.

(2) $\quad N_{\mathcal{C}}(n, d)<\infty$ for $n \geq 1$ and $0 \leq d<\infty$.

(3) For fixed $d<\infty$, we have $\lim _{n \rightarrow \infty} \sup N_{\mathcal{C}}(n, d) \leq 6$.

(4) If $X$ is a compact manifold with boundary and $n \geq 2$, then

$$
\overline{\mathrm{rk}}_{\mathcal{C}}\left(C(X) \otimes M_{n}\right) \geq \frac{\operatorname{dim}(X)-2}{4\left(4 n^{2}-1\right)}-2 .
$$

Proof. Every multiplicative commutator of elements of $\operatorname{inv}_{0}\left(C(X) \otimes M_{n}\right)$ is clearly in $\operatorname{inv}_{0}\left(C(X) \otimes M_{n}\right)$ and has determinant 1 . Thus

$$
P_{\mathcal{C}}\left(C(X) \otimes M_{n}\right) \subset\left\{a \in \operatorname{inv}_{0}\left(C(X) \otimes M_{n}\right): \operatorname{det}(a)=1\right\} .
$$

The rest of part (1), and part (2), follows from [28] as in the proof of the corresponding parts of Theorem 2.3, because every elementary matrix is a commutator of elements of $\operatorname{inv}_{0}\left(C(X) \otimes M_{n}\right)$. (For $n \geq 3$, it is well known that every elementary matrix is actually a commutator of elementary matrices. For $n=2$, see the proof of Theorem 5.4 of [13].)

Part (3) now follows from Theorem 2(d) of [5], using the fact that $\operatorname{sr}\left(C(X) \otimes M_{n}\right)<\infty([\mathbf{2 7}])$.

Part (4) follows from Theorem 5.4 of [13], since the element $u$ there is in $\operatorname{inv}_{0}\left(C(X) \otimes M_{n}\right)$.

We note that part (1) of this result is in Proposition 2.4 of [26]. There is a similar result for products of commutators of arbitrary elements in 
$\operatorname{inv}\left(C(X) \otimes M_{n}\right)$. Unfortunately, in (1) and (2) one must then apparently require

$$
n \geq \operatorname{sr}(C(X))+1=[\operatorname{dim}(X) / 2]+2,
$$

is where [ ] is the greatest integer function.

\section{Factorization of unitaries.}

We start this section with the statement of the factorization theorem for exponentials of skewadjoint elements, essentially proved in [13]. We then prove factorization theorems for ${ }^{*}$-symmetries and for commutators of elements of $U_{0}(A)$. Unfortunately, triangular matrices are unitary only if they are diagonal, so the theorem of Dennis and Vaserstein used in the last section does not help here. We must therefore use more direct methods. (Presumably, these methods could also have been applied to some of the problems considered in the previous section.)

3.1 Theorem. Let $\mathcal{C}$ be the class of exponentials of skewadjoint elements. Then:

(1) $\quad P_{\mathcal{C}}\left(C(X) \otimes M_{n}\right)=\bar{P}_{\mathcal{C}}\left(C(X) \otimes M_{n}\right)=U_{0}\left(C(X) \otimes M_{n}\right)$.

(2) $\quad N_{\mathcal{C}}(n, d)<\infty$ for $n \geq 1$ and $0 \leq d<\infty$.

(3) For fixed $d<\infty$, we have $\lim _{n \rightarrow \infty} \sup N_{\mathcal{C}}(n, d) \leq 4$.

(4) If $X$ is a compact manifold with boundary and $n \geq 2$, then

$$
\overline{\mathrm{rk}}_{\mathcal{C}}\left(C(X) \otimes M_{n}\right) \geq \frac{\operatorname{dim}(X)-2}{n^{2}-1}-1 .
$$

Proof. (1) is well known (see [2, Proposition 3.4.5]), (2) is Corollary 3.2 of [13], (3) is Theorem 3.4 of [13], and (4) is Theorem 2.3 of [13].

As with exponentials of arbitrary elements, we will need a stronger statement than part (4) of this theorem. Its proof is the same as that of Proposition 2.2 , and is omitted.

3.2 Proposition. Let $X$ be a compact manifold with boundary, and let $n \geq$ 2. Let $l$ be the least integer such that $l \geq[\operatorname{dim}(X)-2] /\left[n^{2}-1\right]-1$. Then there is a homotopically trivial $u \in C\left(X, S U_{n}\right)$ which is not a limit of products of fewer then l exponentials of skewadjoint elements of $C(X) \otimes M_{n}$.

3.3 Lemma. (Compare [13, Lemma 3.1].) For integers $n \geq 2$ and $d \geq 0$, there is $M(n, d)<\infty$ such that for every compact metric space $X$ of dimension at most $d$ and every homotopically trivial $u \in C\left(X, S U_{n}\right)$, 
there exists a rectifiable path from $u$ to 1 in $C\left(X, S U_{n}\right)$ with length at most $M(n, d)$.

Proof. Let $\rho$ be the metric induced on $S U_{n}$ by some Riemannian metric (or by some realization of it as a finite simplicial complex). Apply Theorem 0.2 of [3] to obtain a number $b_{d}=b_{d}\left(S U_{n}\right)<\infty$. Choose $M(n, d)$ to be any real number greater than $b_{d}\left(S U_{n}\right)$. Then for every compact metric space $X$ with $\operatorname{dim}(X) \leq d$, and every homotopically trivial $u \in C\left(X, S U_{n}\right)$, there is a homotopy $t \mapsto v_{t}$ from $v_{0}=1$ to $v_{1}=u$ with width [3] less than $M(n, d)$. Corollary 3.6 of [3] gives a Lipschitz homotopy $t \mapsto u_{t}$ with Lipschitz constant less than $M(d, n)$; clearly the length of $t \mapsto u_{t}$ in $C(X) \otimes M_{n}$ is bounded by this Lipschitz constant. (Note that the existence of the geodesic equilocally convex structure on $S U_{n}$, needed implicitly in Corollary 3.6 of [3], is ensured by Lemma 1.2 of [3].)

Part (2) of the following lemma is a quantitative improvement of Lemma 2.2 of [26]. We need only two commutators rather than $n$ of them. (We also get a much larger value of $\varepsilon_{n}$ in the proof.) It is interesting to note that at least part (2) can fail if $C(X) \otimes M_{n}$ is replaced by the section algebra of a locally trivial $M_{n}$-bundle. (We omit the example, since it would take us too far afield.)

3.4 Lemma. For each $n \geq 1$ there exists $\varepsilon_{n}>0$ such that whenever $X$ is a compact space and $u: X \rightarrow S U_{n}$ is continuous and satisfies $\|u-1\|<\varepsilon_{n}$, then:

(1) There exist $6^{*}$-symmetries $s_{1}, \ldots, s_{6} \in C(X) \otimes M_{n}$ such that $u=$ $s_{1} s_{2} s_{3} s_{4} s_{5} s_{6}$.

(2) There exist 4 homotopically trivial elements $w_{1}, w_{2}, w_{3}, w_{4} \in C\left(X, S U_{n}\right)$ such that $u=\left(w_{1} w_{2} w_{1}^{*} w_{2}^{*}\right)\left(w_{3} w_{4} w_{3}^{*} w_{4}^{*}\right)$.

(3) If $p \in C(X) \otimes M_{n}$ is a projection unitarily equivalent to a constant projection, then there exist 2 homotopically trivial elements $w_{1}, w_{2} \in$ $C\left(X, S U_{n}\right)$ such that $u p u^{*}=\left(w_{1} w_{2} w_{1}^{*} w_{2}^{*}\right) p\left(w_{1} w_{2} w_{1}^{*} w_{2}^{*}\right)$.

Proof. Conjugating everything by an appropriate unitary, we may assume in case (3) that $p$ is a constant diagonal projection.

We now suppose $n$ is even. Let $\xi=\exp (\pi / n)$, a primitive $2 n$-th root of 1. Define $u_{0}=\operatorname{diag}\left(\xi, \xi^{3}, \ldots, \xi^{2 n-1}\right) \in S U_{n}$, and let $u_{0} \in C(X) \otimes M_{n}$ also denote the constant function with this value. Let $0<\varepsilon_{n}<|1-\xi|=$ $2 \arcsin (1 /(4 n))$. Then the closed $\varepsilon_{n}$-balls about the eigenvalues of $u_{0}$ have disjoint intersections with $S^{1}$. By Theorem 13.6 of [1] (see Section 11 of [1] for the notation), if $v \in U\left(M_{n}\right)$ and $\left\|v-u_{0}\right\| \leq \varepsilon_{n}$, then the closed $\varepsilon_{n}$-ball about any eigenvalue of $u_{0}$ contains exactly one eigenvalue of $v$. In particular, if $\|u-1\| \leq \varepsilon_{n}$, then $u u_{0}$ has distinct eigenvalues at each point of $X$. We claim 
that there exists $z \in U\left(C(X) \otimes M_{n}\right)$ such that $z u u_{0} z^{*}$ is diagonal. To see this, apply the argument of the second paragraph of the proof of Lemma 2.4 of [11] to the unitary defined on $V=\left\{c \in U\left(M_{n}\right):\left\|c-u_{0}\right\| \leq \varepsilon_{n}\right\}$ by $c \mapsto$ c, to obtain $f: V \rightarrow U\left(M_{n}\right)$ such that $f(c) c f(c)^{*}$ is diagonal for all $c \in V$. Now define $z(x)=f\left(u(x) u_{0}(x)\right)$ for $x \in X$.

Write $z u u_{0} z^{*}=\operatorname{diag}\left(\lambda_{1}, \ldots, \lambda_{n}\right)$ with continuous functions $\lambda_{1}, \ldots, \lambda_{n}$ : $X \rightarrow S^{1}$. Each of these functions has range contained in a ball of radius less than $2 \arcsin (1 / 4)$ centered on the unit circle, and so is homotopically trivial. Furthermore, $\lambda_{1}(x) \cdots \lambda_{n}(x)=1$ for all $x$.

Following the proof of Theorem 3 of [21], we factor $z u u_{0} z^{*}$ continuously as

(*) $\operatorname{diag}\left(\lambda_{1}, \bar{\lambda}_{1}, \lambda_{1} \lambda_{2} \lambda_{3}, \bar{\lambda}_{1} \bar{\lambda}_{2} \bar{\lambda}_{3}, \ldots\right) \operatorname{diag}\left(1, \lambda_{1} \lambda_{2}, \bar{\lambda}_{1} \bar{\lambda}_{2}, \lambda_{1} \lambda_{2} \lambda_{3} \lambda_{4}, \ldots\right)$

(The last diagonal entry of the second factor is $\lambda_{1}(x) \cdots \lambda_{n}(x)=1$.) The equation

$$
\left(\begin{array}{cc}
\alpha(x) & 0 \\
0 & \bar{\alpha}(x)
\end{array}\right)=\left(\begin{array}{cc}
0 & \alpha(x) \\
\bar{\alpha}(x) & 0
\end{array}\right)\left(\begin{array}{ll}
0 & 1 \\
1 & 0
\end{array}\right)
$$

applied to appropriate pairs of entries in $\left({ }^{*}\right)$, shows that each factor in $\left(^{*}\right)$ is a product of two ${ }^{*}$-symmetries. Therefore $u u_{0}$ is a product of four ${ }^{*}$ symmetries. We can further use $\left(^{* *}\right)$ in the same way as above to show that $u_{0}$ is a product of two ${ }^{*}$-symmetries. Then $u=\left(u u_{0}\right) u_{0}^{*}$ is a product of six *-symmetries, proving (1).

To prove (2) and (3), we write

$$
z u u_{0} z^{*}=\operatorname{diag}\left(\lambda_{1}, \ldots, \lambda_{n}\right)=w_{1} w_{2} w_{1}^{*} w_{2}^{*}
$$

with

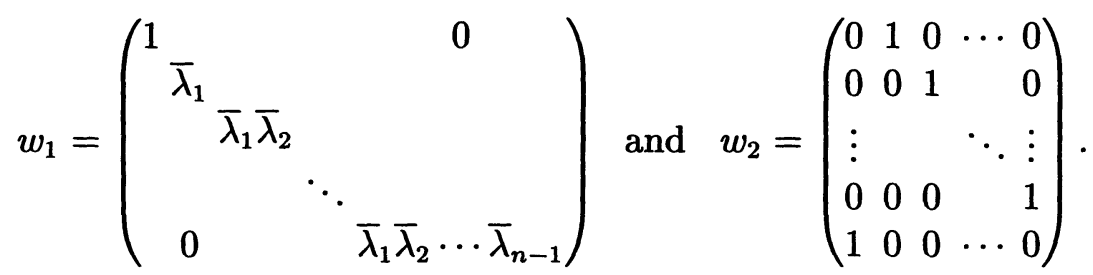

(This works since $\lambda_{1}(x) \cdots \lambda_{n}(x)=1$. Compare with Lemma 2.1 of [26].) Therefore $u u_{0}$ is a commutator. The entries of $w_{1}$ are homotopically trivial. Therefore we may replace $w_{1}$ by $\operatorname{det}\left(w_{1}\right)^{-1 / n} w_{1}$, and similarly for $w_{2}$, to express $u u_{0}$ as a commutator of homotopically trivial functions from $X$ to $S U_{n}$. Our choices imply that $u_{0}$ commutes with $p$. Therefore $\left(u u_{0}\right) p\left(u u_{0}\right)^{*}=$ 
$u p u^{*}$, and we have proved (3). To prove (2), we use the same trick as above to express $u_{0}^{*}$ as a commutator.

If $n$ is odd, we take $\xi=\exp (2 \pi i / n), u_{0}=\operatorname{diag}\left(1, \xi, \ldots, \xi^{n-1}\right)$, and $0<\varepsilon_{n}<|1-\xi|=2 \arcsin (1 /(2 n))$ instead. Define $\lambda_{j}$ as before. The proofs of (2) and (3) (involving commutators) are unchanged. For (1), we use the factorization $\left(^{*}\right)$ as before. This time, each of the two factors of $\left.{ }^{*}\right)$, as well as $u_{0}^{*}$, has diagonal entries that match up in complex conjugate pairs, with one entry left over, which is equal to 1 . Each is therefore again a product of two ${ }^{*}$-symmetries, proving (1).

Combining Lemmas 3.3(1) and 3.4(1) and (2) yields the following result. The part of part (1) which applies to commutators has already been observed by Thomsen (in Proposition 2.4 of [26]).

3.5 Lemma. If $\mathcal{C}$ is either the class of ${ }^{*}$-symmetries, or the class of commutators of homotopically trivial unitaries, then:

(1) $\quad P_{\mathcal{C}}\left(C(X) \otimes M_{n}\right)=\bar{P}_{\mathcal{C}}\left(C(X) \otimes M_{n}\right)$, and is the set of homotopically trivial unitaries, with determinant \pm 1 (depending on $x \in X$ ) for *-symmetries, determinant 1 for commutators.

(2) $N_{\mathcal{C}}(n, d)<\infty$ for $n \geq 1$ and $0 \leq d<\infty$.

Proof. (1) We first observe that commutators of elements of $U_{0}\left(C(X) \otimes M_{n}\right)$, as well as ${ }^{*}$-symmetries, are homotopically trivial, that is, in $U_{0}(C(X) \otimes$ $\left.M_{n}\right)$. An argument similar to one in the proof of Theorem 3.4(1) shows that $\left\{u \in U_{0}\left(C(X) \otimes M_{n}\right): \operatorname{det}(u)=1\right\}$ is the set of homotopically trivial elements of $C\left(X, S U_{n}\right)$, and that

$$
\left\{u \in U_{0}\left(C(X) \otimes M_{n}\right): \operatorname{det}(u(x)) \in\{ \pm 1\} \text { for all } x\right\}
$$

has exactly one path component corresponding to each continuous \pm 1 -valued function on $X$.

The previous lemma implies that the subgroups generated by the commutators and the ${ }^{*}$-symmetries both contain an $\varepsilon$-neighborhood of 1 in $C\left(X, S U_{n}\right)$. Furthermore, if $\lambda: X \rightarrow\{ \pm 1\}$ is continuous, then $\operatorname{diag}(\lambda, 1, \ldots, 1)$ is a ${ }^{*}$-symmetry contained in the corresponding path component of

$$
\left\{u \in U_{0}\left(C(X) \otimes M_{n}\right): \operatorname{det}(u(x)) \in\{ \pm 1\} \text { for all } x\right\} .
$$

The claimed identifications of $P_{c}\left(C(X) \otimes M_{n}\right)$ follow. Since these are closed subgroups of $\operatorname{inv}_{0}\left(C(X) \otimes M_{n}\right)$, we have $P_{C}\left(C(X) \otimes M_{n}\right)=\bar{P}_{C}\left(C(X) \otimes M_{n}\right)$ in both cases.

(2) With $M(n, d)$ as in Lemma 3.3(1) and $\varepsilon_{n}$ as in Lemma 3.4, let $r(n, d)$ be the least integer exceeding $M(n, d) / \varepsilon_{n}$. Then $N_{\mathcal{C}}(n, d) \leq 2 r(n, d)$ is clear 
for commutators. For ${ }^{*}$-symmetries, one extra one suffices to get into any path component of $P_{\mathcal{C}}\left(C(X) \otimes M_{n}\right)$, so $N_{\mathcal{C}}(n, d) \leq 6 r(n, d)+1$.

The previous lemma gives the first two parts of our standard factorization theorem for unitary commutators and for *-symmetries. Unfortunately what we have done so far does not give the third part. It turns out to be true that the numbers $M(n, d)$ of Lemma 3.3 can be chosen to satisfy $\lim _{n \rightarrow \infty} \sup M(n, d) \leq C$ for some fixed $C$ independent of $d$. (See Remark 4.11.) This, however, does not help, since the numbers $\varepsilon_{n}$ in the proof of Lemma 3.4 go to 0 as $n \rightarrow \infty$. Further progress requires another idea, which we take from [15].

3.6 Lemma. Let $A$ be a unital $\mathrm{C}^{*}$-algebra.

(1) Let $p, q, r \in A$ be unitarily equivalent projections with $p$ orthogonal to $q$ and $q$ orthogonal to $r$. Then there exists a commutator $u=z_{1} z_{2} z_{1}^{*} z_{2}^{*}$, with $z_{1}, z_{2} \in U_{0}(A)$, such that upu* $=r$.

(2) Let $u \in U(A)$. Then $u \oplus u^{*} \in U_{0}\left(M_{2}(A)\right)$ is a product of two *symmetries in $M_{2}(A)$, and is also a commutator wvw* $v^{*}$ with $w, v \in U_{0}\left(M_{2}(A)\right)$.

Proof. (1) Let $v$ be a unitary with $v p v^{*}=q$. Define

$$
e=\frac{1}{2}\left(p+q+p v^{*} q+q v p\right) \text { and } w=1-p-q+\frac{1}{\sqrt{2}}\left(p+q-p v^{*} q+q v p\right) .
$$

Calculations show that $e$ is a projection, $w$ is a unitary, $w p w^{*}=e$, and

$$
(1-2 e) p(1-2 e)^{*}=q \text {. }
$$

Furthermore, the unitary path

$$
t \mapsto 1+(\cos (t)-1)(p+q)+\sin (t)\left(q v p-p v^{*} q\right),
$$

for $t \in[0, \pi / 4]$, shows that $w \in U_{0}(A)$.

In the same manner, we can construct a projection $f$ and a unitary $x \in U_{0}(A)$ such that $x q x^{*}=f$ and

$$
(1-2 f) q(1-2 f)^{*}=r \text {. }
$$

Set $u=(1-2 f)(1-2 e)$; combining the previous equation with $\left(^{*}\right)$ yields $u p u^{*}=r$.

Set $z_{2}=w(1-2 e)^{*} x^{*}$. Note that $z_{2} f z_{2}^{*}=e$ by $\left(^{*}\right)$ and the choices of $w$ and $x$. Furthermore, $z_{2} \in U_{0}(A)$ because $w, x \in U_{0}(A)$ and $(1-2 e)^{*}$ is 
a ${ }^{*}$-symmetry. Further set $z_{1}=1-2 f$, which is in $U_{0}(A)$ because it is a *-symmetry. Then

$$
z_{1} z_{2} z_{1}^{*} z_{2}^{*}=(1-2 f)\left(1-2 z_{2} f z_{2}^{*}\right)=(1-2 f)(1-2 e)=u .
$$

So $u$ is a commutator of elements of $U_{0}(A)$.

(2) Define

$$
v=\frac{1}{2}\left(\begin{array}{cc}
1+u^{*} & 1-u^{*} \\
-1+u & 1+u
\end{array}\right)=\frac{1}{\sqrt{2}}\left(\begin{array}{cc}
1 & u^{*} \\
u & -1
\end{array}\right) \cdot \frac{1}{\sqrt{2}}\left(\begin{array}{cc}
1 & 1 \\
1 & -1
\end{array}\right) .
$$

Each factor on the right is easily checked to be ${ }^{*}$-symmetry. Therefore $v \in U_{0}\left(M_{2}(A)\right)$. Now one checks that

$$
\left(\begin{array}{cc}
u & 0 \\
0 & u^{*}
\end{array}\right)=\left(\begin{array}{ll}
0 & 1 \\
1 & 0
\end{array}\right) v\left(\begin{array}{ll}
0 & 1 \\
1 & 0
\end{array}\right)^{*} v^{*}
$$

This exhibits $u \oplus u^{*}$ as a commutator of elements of $U_{0}\left(M_{2}(A)\right)$. Since the product of the last three factors is a conjugate of a ${ }^{*}$-symmetry, it also exhibits $u \oplus u^{*}$ as a product of two ${ }^{*}$-symmetries.

3.7 Lemma. Let $X$ be compact metric, and let $n \geq 5 \operatorname{dim}(X)+3$. If $p, q \in C(X) \otimes M_{n}$ are homotopic projections which are unitarily equivalent to constant projections, then:

(1) There exists $v \in U_{0}\left(C(X) \otimes M_{n}\right)$, a product of at most $5^{*}$-symmetries, such that $v p v^{*}=q$.

(2) There exists $v \in U_{0}\left(C(X) \otimes M_{n}\right)$, a product of at most 3 commutators of elements of $U_{0}\left(C(X) \otimes M_{n}\right)$, such that $v p v^{*}=q$.

Proof. (1) This follows from Theorem 2.1 of [15] and the general relations between the $\mathrm{C}^{*}$ projective length and rank, Theorem 2.4 of [14].

(2) Let $\varepsilon_{n}$ be as in Lemma 3.4. Choose $\delta>0$ such that whenever $e, f \in C\left(X_{k}\right) \otimes M_{n}$ are projections with $\|e-f\|<\delta$, then there exists $u \in U_{0}\left(C(X) \otimes M_{n}\right)$ such that $u e u^{*}=f,\|u-1\|<\varepsilon_{n} / 6$, and $\operatorname{det}(u(x))=1$ for all $x$. (A standard construction produces $\delta$ such that there is always a $u$ satisfying all but the determinant condition. But if $u$ is close enough to 1 , with the required estimate depending only on $n$, one can construct $\operatorname{det}(u)^{-1 / n} u$, and it will still be close to 1.)

Let $p, q \in C(X) \otimes M_{n}$ be homotopic projections. Write $C(X) \otimes M_{n}=$ $\lim _{\rightarrow k} C\left(X_{k}\right) \otimes M_{n}$, where the $X_{k}$ are finite complexes of dimension at most $\overrightarrow{\operatorname{dim}}(X)$, as in Lemma 1.8 of [15]. As the proof of Proposition 2.11 of [14], we can select a suitable term $C(Y) \otimes M_{n}$ from this direct system, and projections 
$p_{0}, q_{0} \in C(Y) \otimes M_{n}$, such that if $\varphi: C(Y) \otimes M_{n} \rightarrow C(X) \otimes M_{n}$ is the map to the direct limit, then $\left\|p-\varphi\left(p_{0}\right)\right\|,\left\|q-\varphi\left(q_{0}\right)\right\|<\delta$. Choosing $C(Y) \otimes M_{n}$ to be sufficiently far out in the direct system, we may further assume that $p_{0}$ and $q_{0}$ are homotopic.

We now work in $C(Y) \otimes M_{n}$. We consider one component of $Y$ at a time; thus, we may assume $Y$ is connected. Then $p_{0}$ and $q_{0}$ have constant rank. Replacing them by $1-p_{0}$ and $1-q_{0}$ if necessary, we assume they have rank at most $n / 2$.

We follow the proof of Theorem 2.1 of [15], with $\Gamma(V)=C(Y) \otimes M_{n}$, and with $p_{0}$ and $q_{0}$ in place of $p$ and $q$. Choose $p_{1}, p_{2}, q_{1}, q_{2}$ as in the second half of that proof, with ranks as chosen in the first half. Choose $\bar{p}_{1}$ and $\bar{q}_{1}$ as there, with $\left\|\bar{p}_{1}-p_{1}\right\|,\left\|\bar{q}_{1}-q_{1}\right\|<\delta$. Further choose $f_{1}$ as there. Then $\bar{p}_{1}, \bar{q}_{1}$, and $f_{1}$ are equivalent projections with $\bar{p}_{1}$ orthogonal to $f_{1}$ and $f_{1}$ orthogonal to $\bar{q}_{1}$. Lemma 3.6 (1) therefore provides a commutator $z_{1}$ such that $z_{1} \bar{p}_{1} z_{1}^{*}=\bar{q}_{1}$. The first paragraph of the proof provides unitaries $c_{1}$ and $c_{2}$ such that $\left\|c_{i}-1\right\|<\varepsilon_{n} / 6$ and $c_{1} \bar{p}_{1} c_{1}^{*}=p_{1}, c_{2} \bar{q}_{1} c_{2}^{*}=q_{1}$. Then $\left(c_{2} z_{1} c_{1}^{*}\right) p_{1}\left(c_{2} z_{1} c_{1}^{*}\right)^{*}=q_{1}$.

Continuing to follow the proof of Theorem 2.1 of [15], let

$$
A=\left(1-q_{1}\right)\left[C(Y) \otimes M_{n}\right]\left(1-q_{1}\right) .
$$

Choose projections $\bar{p}_{2}, \bar{q}_{2} \in A$ as there, with $\left\|\bar{p}_{2}-\left(c_{2} z_{2} c_{1}^{*}\right) p_{2}\left(c_{2} z_{1} c_{1}^{*}\right)^{*}\right\|$, $\left\|\bar{q}_{2}-q_{2}\right\|<\delta$. Choosing $f_{2}$ as there, and applying Lemma 3.6 again, we obtain a commutator $w \in A$ such that $w \bar{p}_{2} w^{*}=\bar{q}_{2}$. Let $z_{2}=q_{1}+w$, again a commutator of homotopically trivial unitaries. Further choose, again as in the first paragraph, unitaries $c_{3}$ and $c_{4}$ such that $\left\|c_{i}-1\right\|<\varepsilon_{n} / 6$ and

$$
c_{3}\left(q_{1}+\bar{p}_{2}\right) c_{3}^{*}=q_{1}+\left(c_{2} z_{1} c_{1}^{*}\right) p_{2}\left(c_{2} z_{1} c_{1}^{*}\right)^{*} \quad \text { and } \quad c_{4}\left(q_{1}+\bar{q}_{2}\right) c_{4}^{*}=q_{1}+q_{2} .
$$

Then $u=c_{4} z_{2} c_{3}^{*} c_{2} z_{1} c_{1}^{*}$ satisfies

$$
u p_{0} u^{*}=u\left(p_{1}+p_{2}\right) u^{*}=q_{1}+q_{2}=q_{0} .
$$

Combining the choice made in the second paragraph of the proof with the result of the first paragraph, we can also find $c_{5}, c_{6} \in U_{0}\left(C(X) \otimes M_{n}\right)$ such that

$$
c_{5} \varphi\left(p_{0}\right) c_{5}^{*}=p \quad \text { and } \quad c_{6} \varphi\left(q_{0}\right) c_{6}^{*}=q .
$$

Set $v_{0}=c_{6} \varphi(u) c_{5}^{*}$. Then $v_{0} p v_{0}^{*}=q$. We can furthermore write

$$
\begin{array}{r}
v_{0}=\left[c_{6} \varphi\left(c_{4} c_{3}^{*} c_{2} c_{1}^{*}\right) c_{5}^{*}\right]\left[\left(c_{5} \varphi\left(c_{1} c_{2}^{*} c_{3}\right)\right) \varphi\left(z_{2}\right)\left(c_{5} \varphi\left(c_{1} c_{2}^{*} c_{3}\right)\right)^{*}\right] \\
{\left[\left(c_{5} \varphi\left(c_{1}\right)\right) \varphi\left(z_{1}\right)\left(c_{5} \varphi\left(c_{1}\right)\right)^{*}\right] .}
\end{array}
$$


Call the factors $s, v_{2}$, and $v_{3}$. Then $v_{2}$ and $v_{3}$ are commutators of homotopically trivial unitaries because $z_{2}$ and $z_{1}$ are. Furthermore, $\|s-1\|<\varepsilon_{n}$, since $\left\|c_{i}-1\right\|<\varepsilon_{n} / 6$ for $i=1, \ldots, 6$. Lemma 3.4(3) therefore provides a commutator $v_{1}$ of homotopically trivial unitaries such that $v_{1}\left(v_{2} v_{3} p v_{3}^{*} v_{2}^{*}\right) v_{1}^{*}=$ $s\left(v_{2} v_{3} p v_{3}^{*} v_{2}^{*}\right) s^{*}=q$. (Note that $p$ is unitarily equivalent to a constant projection.) Therefore $v=v_{1} v_{2} v_{3}$ is the desired product of three commutators.

Let $\langle\alpha\rangle$ denote the least integer $l$ such that $l \geq \alpha$.

3.8 Lemma. Let $n \geq 5 \operatorname{dim}(X)+3$. Let $\mathcal{C}_{1}$ be the class of ${ }^{*}$-symmetries and let $\mathcal{C}_{2}$ be the class of commutators of elements of $U_{0}(A)$. Let $b_{i}=$ $\mathrm{rk}_{\mathcal{C}_{i}}\left(C(X) \otimes M_{n}\right)$. Let $2 n \leq k \leq 4 n$. Then

$$
\operatorname{rk}_{\mathcal{C}_{1}}\left(C(X) \otimes M_{k}\right) \leq 14+\left\langle b_{1} / 2\right\rangle \quad \text { and } \quad \operatorname{rk}_{\mathcal{C}_{2}}\left(C(X) \otimes M_{k}\right) \leq 8+\left\langle b_{2} / 2\right\rangle \text {. }
$$

If $k=2 n$, then

$$
\mathrm{rk}_{\mathcal{C}_{1}}\left(C(X) \otimes M_{k}\right) \leq 7+\left\langle b_{1} / 2\right\rangle \quad \text { and } \quad \operatorname{rk}_{\mathcal{C}_{2}}\left(C(X) \otimes M_{k}\right) \leq 4+\left\langle b_{2} / 2\right\rangle .
$$

Proof. We follow the ideas of Section 3 of [15]. Let $u \in U_{0}\left(C(X) \otimes M_{k}\right)$. Choose orthogonal projections $p_{1}, q_{1}, p_{2}$, and $q_{2} \in M_{k}$ which sum to 1 and such that $\operatorname{rank}\left(p_{i}\right)=n$ and $0 \leq \operatorname{rank}\left(q_{i}\right) \leq n$. Let $p_{i}, q_{i}$ also denote the corresponding constant functions in $C(X) \otimes M_{k}$. By Lemma 3.7, there exists $v_{0} \in C(X) \otimes M_{k}$, either a product of $5^{*}$-symmetries or of 3 commutators, such that $v_{0}^{*}\left(p_{1}+q_{1}\right) v_{0}=u^{*}\left(p_{1}+q_{1}\right) u$, that is, $u v_{0}^{*}$ commutes with $p_{1}+q_{1}$. There further exists $v_{1}$, either a product of $5^{*}$-symmetries or of 3 commutators, of the form $z_{1} \oplus z_{2}$ with $z_{i} \in A_{i}=\left(p_{i}+q_{i}\right)\left(C(X) \otimes M_{k}\right)\left(p_{i}+q_{i}\right)$, such that $u v_{0}^{*} v_{1}^{*}$ comutes with each $p_{i}$ and each $q_{i}$.

We now find $v_{2}$, a product of two ${ }^{*}$-symmetries and also a commutator, such that $u v_{0}^{*} v_{1}^{*} v_{2}^{*}$ still commutes with each $p_{i}$ and each $q_{i}$, and such that $q_{i}\left(u v_{0}^{*} v_{1}^{*} v_{2}^{*}\right) q_{i}=q_{i}$. Again, $v_{2}=z_{1} \oplus z_{2}$ with $z_{i} \in A_{i}$. To construct $z_{i}$, let $r_{i} \leq p_{i}$ be a constant projection with the same rank as $q_{i}$. With respect to the decomposition of the identity $\left(1-r_{i}\right)+r_{i}+q_{i}$ in $A_{i}$, we have the matrix representation

$$
\left(p_{i}+q_{i}\right)\left(u v_{0}^{*} v_{1}^{*}\right)\left(p_{i}+q_{i}\right)=\left(\begin{array}{ccc}
c_{11} & c_{12} & 0 \\
c_{21} & c_{22} & 0 \\
0 & 0 & w
\end{array}\right)
$$

and we take

$$
z_{i}=\left(\begin{array}{ccc}
1 & 0 & 0 \\
0 & w^{*} & 0 \\
0 & 0 & w
\end{array}\right)
$$


By Lemma $3.6(2), z_{i}$ is a commutator of homotopically trivial unitaries, and also a product of two *-symmetries.

The remainder of the proof takes place entirely within

$$
\left(p_{1}+p_{2}\right)\left(C(X) \otimes M_{k}\right)\left(p_{1}+p_{2}\right) \cong M_{2}\left(C(X) \otimes M_{n}\right) .
$$

We write the elements as $2 \times 2$ matrices; elements of $C(X) \otimes M_{k}$ are gotten by adding $q_{1}+q_{2}$ to everything. We know that deleting the summand $q_{1}+q_{2}$ from $u v_{0}^{*} v_{1}^{*} v_{2}^{*}$ leaves a unitary $u_{0}$ of the form

$$
u_{0}=\left(\begin{array}{cc}
s_{1} & 0 \\
0 & s_{2}
\end{array}\right) \text {. }
$$

Note that $\left(s_{1} s_{2} \oplus 1\right)+q_{1}+q_{2} \in U_{0}\left(C(X) \otimes M_{k}\right)$ since it is homotopic to $u$. The topological stable rank $\operatorname{tsr}(C(X))$ is certainly less than $5 \operatorname{dim}(X)+3$, by Proposition 1.7 of [22], and so $s_{1} s_{2} \in U_{0}\left(C(X) \otimes M_{n}\right)$ by Theorem 2.10 of [23]. Furthermore,

$$
\operatorname{det}\left(s_{1} s_{2}\right)=\operatorname{det}\left(\begin{array}{cc}
s_{1} s_{2} & 0 \\
0 & 1
\end{array}\right)=\operatorname{det}\left(u_{0}\right) \operatorname{det}\left(\begin{array}{cc}
s_{2} & 0 \\
0 & s_{2}^{*}
\end{array}\right)=\operatorname{det}\left(u_{0}\right) .
$$

We now assume that $\operatorname{det}(u(x)) \in\{ \pm 1\}$ for all $x$ (in the ${ }^{*}$-symmetry case) or $\operatorname{det}(u)=1$ (in the commutator case). Because the $v_{j}$ are products of ${ }^{*}$-symmetries or commutators, it follows that $\operatorname{det}\left(u v_{0}^{*} v_{1}^{*} v_{2}^{*}\right)$, and so by the above $\operatorname{det}\left(u_{0}\right)$ and $\operatorname{det}\left(s_{1} s_{2}\right)$, are \pm 1 (in the ${ }^{*}$-symmetry case) or 1 (in the commutator case). Therefore $s_{1} s_{2}$ is a product of at most $b_{1}{ }^{*}$-symmetries or $b_{2}$ commutators, and we can write $s_{1} s_{2}=w_{1} w_{2}$, where each $w_{i}$ is a product of at most $\left\langle b_{1} / 2\right\rangle^{*}$-symmetries or $\left\langle b_{2} / 2\right\rangle$ commutators. One checks that $s_{1} s_{2}=w_{1} w_{2}$ implies $u_{0}=v_{4} v_{3}$, where

$$
v_{4}=\left(\begin{array}{cc}
w_{1}^{*} s_{1} & 0 \\
0 & s_{1}^{*} w_{1}
\end{array}\right)=\left(\begin{array}{cc}
w_{1}^{*} s_{1} & 0 \\
0 & \left(w_{1}^{*} s_{1}\right)^{*}
\end{array}\right)
$$

is a product of two *-symmetries and a also commutator of homotopically trivial unitaries by Lemma $3.6(2)$, and

$$
v_{3}=\left(\begin{array}{cc}
s_{1}^{*} w_{1} s_{1} & 0 \\
0 & w_{2}
\end{array}\right)
$$

is a product of $\left\langle b_{1} / 2\right\rangle^{*}$-symmetries or $\left\langle b_{2} / 2\right\rangle$ commutators.

Replacing $v_{j}$ by $v_{j}+q_{1}+q_{2}$ for $j=3$, we get $u=v_{4} v_{3} v_{2} v_{1} v_{0}$, which is a product of $14+\left\langle b_{1} / 2\right\rangle^{*}$-symmetries or of $8+\left\langle b_{2} / 2\right\rangle$ commutators of elements of $U_{0}\left(C(X) \otimes M_{k}\right)$. 
If $k=2 n$ then $q_{1}=q_{2}=0$, and we can take $v_{1}=v_{2}=1$. This leaves only $7+\left\langle b_{1} / 2\right\rangle{ }^{*}$-symmetries or $4+\left\langle b_{2} / 2\right\rangle$ commutators.

3.9 Theorem. Let $\mathcal{C}$ be the class of ${ }^{*}$-symmetries. Then:

(1) $P_{\mathcal{C}}\left(C(X) \otimes M_{n}\right)=\bar{P}_{\mathcal{C}}\left(C(X) \otimes M_{n}\right)=\left\{a \in U_{0}\left(C(X) \otimes M_{n}\right): \operatorname{det}(u(x)) \in\right.$ $\{ \pm 1\}$ for all $x\}$.

(2) $N_{\mathcal{C}}(n, d)<\infty$ for $n \geq 1$ and $0 \leq d<\infty$.

(3) For fixed $d<\infty$, we have $\lim _{n \rightarrow \infty} \sup N_{\mathcal{C}}(n, d) \leq 22$.

(4) If $X$ is a compact manifold with boundary, then

$$
\overline{\mathrm{rk}}_{\mathcal{C}}\left(C(X) \otimes M_{n}\right) \geq 2 \frac{\operatorname{dim}(X)-2}{n^{2}-1}-3
$$

Proof. Parts (1) and (2) are contained in Lemma 3.5. For part (3), fix $d$, and set $b=N_{\mathcal{C}}(5 d+3, d)$, which is finite by (2). Define a sequence of integers $b_{r}$ inductively by $b_{0}=b$ and $b_{r+1}=7+\langle b / 2\rangle$. If $b_{r}>15$ then $b_{r+1}<b$; therefore, there exists $r$ such that $b_{r} \leq 15$. Furthermore, $b_{s} \leq 15$ for all $s \geq r$. Using induction and Lemma 3.8 , we get $N_{\mathcal{C}}\left(2^{s}(5 d+3), d\right) \leq 15$ for all $s \geq r$. Now let $k \geq 2^{r+1}(5 d+3)$. Then there exists $s \geq r$ such that $2 \cdot 2^{s}(5 d+3) \leq k \leq 4 \cdot 2^{s}(5 d+3)$, and Lemma 3.8 shows

$$
N_{\mathcal{C}}(k, d) \leq 14+\left\langle N_{\mathcal{C}}\left(2^{s}(5 d+3), d\right) / 2\right\rangle \leq 22 .
$$

For part (4), we claim that a product of two *-symmetries is a limit of exponentials of skewadjoint elements. The result will then follow from Proposition 3.2. To prove the claim, let $s_{1}$ and $s_{2}$ be ${ }^{*}$-symmetries. Then $i s_{1}$ and $-i s_{2}$ have spectrum contained in $\{ \pm i\}$, and so can be written $i s_{1}=\exp \left(i h_{1}\right)$ and $-i s_{2}=\exp \left(i h_{2}\right)$ with $h_{1}, h_{2}$ selfadjoint and $\left\|h_{1}\right\|=\left\|h_{2}\right\|=\pi / 2$. It follows from Corollary 2.2 of [24] that $\exp \left(i h_{1}\right) \exp \left((1-\varepsilon) i h_{2}\right)=\exp \left(i h_{\varepsilon}\right)$ for some selfadjoint $h_{\varepsilon} \in A$, and clearly $\exp \left(i h_{\varepsilon}\right) \rightarrow\left(i s_{1}\right)\left(-i s_{2}\right)=s_{1} s_{2}$ as $\varepsilon \rightarrow 0$.

3.10 Theorem. Let $\mathcal{C}$ be the class of commutators of homotopically trivial unitaries. Then:

(1) $\quad P_{\mathcal{C}}\left(C(X) \otimes M_{n}\right)=\bar{P}_{\mathcal{C}}\left(C(X) \otimes M_{n}\right)=\left\{a \in U_{0}\left(C(X) \otimes M_{n}\right): \operatorname{det}(u)=\right.$ $1\}$.

(2) $N_{C}(n, d)<\infty$ for $n \geq 1$ and $0 \leq d<\infty$.

(3) For fixed $d<\infty$, we have $\lim _{n \rightarrow \infty} \sup N_{\mathcal{C}}(n, d) \leq 13$.

(4) If $X$ is a compact manifold with boundary, then

$$
\overline{\operatorname{rk}}_{\mathcal{C}}\left(C(X) \otimes M_{n}\right) \geq \frac{\operatorname{dim}(X)-2}{3\left(4 n^{2}-1\right)}-1 .
$$


Proof. Parts (1) and (2) are contained in Lemma 3.5. The argument for Theorem 3.9(3) applies here as well, with different numbers. Since $b>9$ implies $4+\langle b / 2\rangle<b$, we get

$$
\lim _{n \rightarrow \infty} \sup N_{\mathcal{C}}(n, d) \leq 8+\langle 9 / 2\rangle=13 .
$$

For (4), we use the unitary version of Theorem 5.4 of [13]. It asserts that if $n \geq 2$, and $X$ is a compact manifold with boundary of dimension at least $m=6\left(4 n^{2}-1\right) l+2$, then there is $u_{0} \in U_{0}\left(C(X) \otimes M_{n}\right)$ which is a product of unitary commutators but not of $2 l$ or fewer of them. To prove it, observe that, as in [13], we may take $X$ to be the closed unit ball of $\mathbb{R}^{m}$. We use the same $u_{0}$ as in the proof of Theorem 5.4 of [13]. It is a product of unitary commutators by (1). Suppose it were a product of $2 l$ of them. The formula

$$
\left(\begin{array}{cc}
v w v^{*} & 0 \\
0 & 1
\end{array}\right)=\left(\begin{array}{cc}
v w & 0 \\
0 & (v w)^{*}
\end{array}\right)\left(\begin{array}{cc}
v^{*} & 0 \\
0 & v
\end{array}\right)\left(\begin{array}{cc}
w^{*} & 0 \\
0 & w
\end{array}\right)
$$

would then show that $u=u_{0} \oplus 1 \in C(X) \otimes M_{2 n}$ is a product of $6 l$ unitaries of the form $z \oplus z^{*}$. Each of these is a limit of exponentials of skewadjoint elements by Corollary 5 of [12]. So $u$ would be a limit of products of $6 l$ exponentials of skewadjoint elements, contradicting Theorem 1.10 of [13].

3.11 Remark. It seems very unlikely that $N_{\mathcal{C}}(n, d)$ is smaller when $n$ is divisible by a large power of 2 than for other large $n$, when $\mathcal{C}$ is either the class of *-symmetries or of unitary commutators. Therefore we expect that one should be able to replace 22 by 15 in Theorem $3.9(3)$ and 13 by 9 in Theorem 3.10(3).

\section{Factorization problems related to exponential length.}

In this section we prove factorization theorems for the class $\mathcal{S}$ of positivestable elements, the class $\mathcal{A}$ of accretive elements, and the class $\mathcal{U}$ of accretive unitaries. The most obvious formulations of these factorization problems yield behavior like that of the $\mathrm{C}^{*}$ exponential length $\operatorname{cel}(A)$ introduced in [24]. (See Theorem 4.6.) However, restriction to elements of $C(X) \otimes \bar{M}_{n}$ with determinant 1 , a subset intrinsically characterized as the commutator subgroup, yields factorization theorems of the same sort as in the previous two sections. (See Theorems 4.12, 4.13, and 4.14.) Indeed, in Theorem 4.10 we see that, under this restriction, the $C^{*}$ exponential length itself behaves in the same way. 
We point out that $\operatorname{rk}_{\mathcal{S}}(A)$ can be sensibly defined for Banach algebras $A$. Its behavior, as illustrated in Proposition 4.5 and Theorem 4.6, suggests that it is related to the Banach exponential rank in somewhat the same way that $\mathrm{C}^{*}$ exponential length is related to $\mathrm{C}^{*}$ exponential rank.

We begin this section with a few lemmas about the classes $\mathcal{S}, \mathcal{A}$, and $\mathcal{U}$. They are probably known, but we lack a reference. We next relate the associated ranks to $\operatorname{cel}(A)$, and prove the theorems for the unrestricted factorization problems. We then introduce the notation for the restricted problems, and prove the corresponding theorems.

Recall that in Definition $1.1(5)$, we defined a to be accretive if $\left(a+a^{*}\right) / 2$ is positive and invertible. This definition agrees with [29] but, as the following lemma shows, not with other commonly used definitions.

4.1 Lemma. Let $H$ be a Hilbert space, and let $a \in L(H)$. Then a is accretive if and only if there is $\varepsilon>0$ such that the numerical range $W(a)$ is contained in $\{\lambda \in \mathbb{C}: \operatorname{Re}(\lambda) \geq \varepsilon\}$.

Proof. Recall ([8, Chapter 17]) that

$$
W(a)=\{\langle a \xi, \xi\rangle: \xi \in H,\|\xi\|=1\} .
$$

Let $a=b+i c$ with $b, c$ selfadjoint. Then clearly

$$
\{\operatorname{Re}(\lambda): \lambda \in W(a)\}=W(b),
$$

and it is well known that a selfadjoint $b \in L(H)$ is positive and invertible if and only if there is $\varepsilon>0$ such that $\langle b \xi, \xi\rangle \geq \varepsilon\|\xi\|^{2}$ for $\xi \in H$.

At this point, we can contrast our definition with the more conventional definitions of accretive operators on Hilbert spaces which are used in the theory of semigroups. In Section V.3.10 of Kato's book [9], a not necessarily bounded operator $a$ is called accretive essentially when

$$
W(a) \subset\{\lambda \in \mathrm{C}: \operatorname{Re}(\lambda) \geq 0\} .
$$

Fillmore ([7], page 87) uses a different sign convention in his semigroups, and therefore requires $W(a) \subset\{\lambda \in \mathrm{C}: \operatorname{Re}(\lambda) \leq 0\}$. Note that both definitions apply to unbounded operators, which we do not have in $\mathrm{C}^{*}$-algebras, and that even Kato's definition does not reduce to that of [29] for finite dimensional Hilbert spaces.

4.2 Corollary. Let $A$ be a unital $\mathrm{C}^{*}$-algebra. Then $a \in A$ is accretive if and only if $\operatorname{Re}(\varphi(a))>0$ for every state $\varphi$ on $A$.

Proof. Note that if $a$ is accretive and $\pi$ is a unital homomorphism, then $\pi(a)$ is accretive. So if $a \in A$ is accretive and $\varphi$ is a state, we show $\operatorname{Re}(\varphi(a))>0$ 
by applying the Gelfand-Naimark-Segal construction to $\varphi$. Conversely, if $a$ is not accretive, there is a state $\varphi$ such that $\varphi\left(\left(a+a^{*}\right) / 2\right) \leq 0$. Then $\operatorname{Re}(\varphi(a)) \leq 0$.

4.3 Corollary. Let $A$ be a unital $\mathrm{C}^{*}$-algebra. If $a \in A$ is accretive, then a is positive-stable, and, in particular, invertible.

Proof. Represent $A$ faithfully on a Hilbert space, and use the lemma together with the fact $([8$, Problem 169]) that for $a \in L(H)$ we have $\operatorname{sp}(a) \subset$ $\bar{W}(a)$.

4.4 Lemma. Let $A$ be a unital $\mathrm{C}^{*}$-algebra, and let $u \in U(A)$. Then the following are equivalent:

(1) $u$ is accretive.

(2) $u$ is positive-stable.

(3) $\|u-1\|<\sqrt{2}$.

(4) $\operatorname{cel}(u)<\pi / 2$.

Proof. (1) $\Leftrightarrow(2)$ : Represent $A$ faithfully on a Hilbert space, and use the fact that for normal $a \in L(H)$, the convex hull of $\operatorname{sp}(a)$ is $\overline{W(a)}$ ([8, Problem 171]).

(2) $\Leftrightarrow(3)$ : Clearly $u$ is positive-stable if and only if $\operatorname{sp}(u)$ is contained in $S^{1} \cap\{\lambda \in \mathbb{C}: \operatorname{Re}(\lambda)>0\}$. Now use the fact that the norm of $u-1$ is equal to its spectral radius.

(3) $\Leftrightarrow(4)$ : See Proposition 2.4 of [24].

This lemma implies that $\operatorname{rk}_{\mathcal{U}}(A)$ is essentially a discrete version of the $\mathrm{C}^{*}$ exponential length. The precise statement is:

$$
\overline{\mathrm{rk}}_{\mathcal{U}}(A)=m \quad \text { if and only if } \quad \operatorname{cel}(A) \in(\pi(m-1) / 2, \pi m / 2] .
$$

(Compare with part (2) of the next proposition.)

Because of this relation, we would like to propose $\operatorname{rk}_{\mathcal{S}}(A)$ as the appropriate analog for Banach algebras of the $C^{*}$ exponential length. The direct analog is infinite even for $M_{2}$, as one sees by considering the matrix $\left(\begin{array}{ll}1 & \alpha \\ 0 & 1\end{array}\right)$ for $|\alpha|$ large. It doesn't help to restrict to elements of determinant 1. On the other hand, the definition of $\operatorname{rk}_{\mathcal{S}}(A)$ makes sense for unital Banach algebras $A$. We will see in the next proposition that $\operatorname{rk}_{\mathcal{S}}(A) \leq \operatorname{rk}_{\mathcal{U}}(A)+1$ if $A$ is a $\mathrm{C}^{*}$-algebra, just as $\operatorname{ber}(A) \leq \operatorname{cer}(A)+1$. In the theorem following, we will also see that it behaves like cel on the algebras $C(X) \otimes M_{n}$, for which we already know that the behavior of ber is similar to that of cer.

4.5 Proposition. Let $A$ be any unital $\mathrm{C}^{*}$-algebra. Then:

(1) $P_{\mathcal{A}}(A)=\bar{P}_{\mathcal{A}}(A)=P_{\mathcal{S}}(A)=\bar{P}_{\mathcal{S}}(A)=\operatorname{inv}_{0}(A)$ and $P_{\mathcal{U}}(A)=\bar{P}_{\mathcal{U}}(A)=$ $U_{0}(A)$. 
(2) $\operatorname{rk}_{\mathcal{S}}(A) \leq \overline{\mathrm{rk}}_{\mathcal{A}}(A)+1, \operatorname{rk}_{\mathcal{A}}(A) \leq \overline{\mathrm{rk}}_{\mathcal{A}}(A)+1$, and $\mathrm{rk}_{\mathcal{U}}(A) \leq \overline{\mathrm{rk}}_{\mathcal{U}}(A)+1$.

(3) $\operatorname{rk}_{\mathcal{S}}(A) \leq \overline{\operatorname{rk}}_{\mathcal{A}}(A) \leq \operatorname{rk}_{\mathcal{U}}(A)+1 \leq 2 \operatorname{cel}(A) / \pi+2$ and $\operatorname{cel}(A) \leq \pi \operatorname{rk}_{\mathcal{U}}(A) / 2$.

Proof. (1) The sets of accretive and positive-stable elements of $A$ are both contained in $\operatorname{inv}_{0}(A)$ (in fact, in $\exp (A)$ ) and both contain neighborhoods of 1 in $A$. Similarly, the set of accretive unitaries is contained in $U_{0}(A)$ and contains a neighborhood of 1 in $U(A)$.

(2) This follows from the same openness properties as in the proof of (1).

(3) The first inequality is trivial. The second follows from polar decomposition. The third follows from the implication $(4) \Rightarrow(1)$ of the previous lemma, by choosing appropriate break points on a unitary path from 1 to $u \in U_{0}(A)$ of length at most $\operatorname{cel}(u)+\varepsilon$, and because the accretive unitaries contain a neighborhood of 1 in $U_{0}(A)$. The last inequality follows from the implication $(1) \Rightarrow(4)$ of the previous lemma.

4.6 Theorem. Let $X$ be compact metric, let $n \geq 1$, and let $A=C(X) \otimes M_{n}$.

(1) If $X$ is totally disconnected, then $\operatorname{rk}_{\mathcal{S}}(A), \operatorname{rk}_{\mathcal{A}}(A) \leq 4, \operatorname{rk}_{\mathcal{U}}(A) \leq 3$, and $\operatorname{cel}(A) \leq \pi$.

(2) If $X$ is not totally disconnected, then $\overline{\mathrm{rk}}_{\mathcal{S}}(A)=\overline{\mathrm{rk}}_{\mathcal{A}}(A)=\overline{\mathrm{rk}}_{\mathcal{U}}(A)=$ $\operatorname{cel}(A)=\infty$.

Proof. (1) If $X$ is totally disconnected, then any unitary in $A$ can be approximated arbitrarily closely by unitary functions having only finitely many values. So clearly $\operatorname{cel}(A) \leq \pi$. The rest now follows from the previous proposition.

(2) In view of the previous proposition, it suffices to show that $\operatorname{rk}_{\mathcal{S}}(A)=$ $\infty$. Since $X$ is not totally disconnected, there exist distinct $x_{1}, x_{2} \in X$ such that every closed and open subset of $X$ which contains $x_{1}$ also contains $x_{2}$. Choose a continuous function $f: X \rightarrow \mathbb{R}$ such that $f\left(x_{1}\right)=1$ and $f\left(x_{2}\right)=-1$. Define $u \in C(X) \otimes M_{n}$ by $u=\exp (i M f) \otimes 1$, where $M$ is some positive real number. We will show that if $M>\pi l / 2$, then $u$ is not a limit of products of $l$ positive-stable elements of $A$.

Suppose $a$ is a product of $l$ positive-stable elements of $A$, that is, $a=$ $a_{1} \cdots a_{l}$ with $a_{j}$ positive-stable. Note that $1-t+t a_{j}$ is also positive-stable for $t \in[0,1]$. Define

$$
b(t)=\prod_{j=1}^{l}\left(1-t+t a_{j}\right) .
$$

We now calculate the continuous $\operatorname{logarithm} \log (\operatorname{det}(b(t)))$ which is 0 when $t=0$. Functional calculus with logarithms is taken with the continuous branch which is real on positive real numbers and undefined on negative real 
numbers. We have

$$
\log (\operatorname{det}(b(t)))=\sum_{j=1}^{l} \log \left(\operatorname{det}\left(1-t+t a_{j}\right)\right)=\sum_{j=1}^{l} \operatorname{tr}\left(\log \left(1-t+t a_{j}\right)\right) .
$$

The second equality holds because both sides are continuous and agree when $t=0$. Each term in the last sum has imaginary part in $[\pi n / 2, \pi n / 2]$. Therefore, putting $t=1$, we get

$$
|\operatorname{Im}(\log (\operatorname{det}(a)))| \leq \pi n l / 2 .
$$

One easily checks that even if $a$ is only a limit of products of $l$ positive-stable elements, its determinant must still have a logarithm satisfying $\left({ }^{*}\right)$. On the other hand, if $f$ is a logarithm of $\operatorname{det}(u)$, then $f\left(x_{1}\right)-f\left(x_{2}\right)=2 M n i$. So $u$ cannot be a limit of products of $l$ positive-stable elements.

The previous theorem shows that, at least on $C(X) \otimes M_{n}$, the quantities $\mathrm{rk}_{\mathcal{A}}$ and $\mathrm{rk}_{\mathcal{S}}$ also behave like $\mathrm{C}^{*}$ exponential length. Nevertheless, behavior like that of exponential rank is hidden just beneath the surface. (This is even true for the $\mathrm{C}^{*}$ exponential length itself.) To expose it, we restrict to factorizations of the elements of determinant 1 , intrinsically characterized as the commutator subgroup. (See the characterizations of the commutator subgroups of $\operatorname{inv}_{0}(A)$ and $U_{0}(A)$ in Theorems 2.10(1) and 3.10(1).)

4.7 Definition. Let $\mathcal{C}$ be one of the clases $\mathcal{A}, \mathcal{S}$, or $\mathcal{U}$. For a topological group $G$ we let $G^{\prime}$ denote the closed subgroup generated by the commutators. Let $A$ be a unital $\mathrm{C}^{*}$-algebra, and define:

(1) $P_{\mathcal{C}}^{\prime}(A)=P_{\mathcal{C}}(A) \cap \operatorname{inv}_{0}(A)^{\prime}$ if $\mathcal{C}=\mathcal{A}$ or $\mathcal{S}$, and $P_{\mathcal{C}}^{\prime}(A)=P_{\mathcal{C}}(A) \cap U_{0}(A)^{\prime}$ if $\mathcal{C}=\mathcal{U}$.

(2) $\bar{P}_{\mathcal{C}}^{\prime}(A)$ is the closure of $P_{\mathcal{C}}^{\prime}(A)$ in $\operatorname{inv}_{0}(A)$.

(3) $\operatorname{rk}_{\mathcal{C}}^{\prime}(A)$ is the smallest $n$ (possibly $\infty$ ) such that every element of $P_{\mathcal{C}}^{\prime}(A)$ is a product of at most $n$ elements of the class $\mathcal{C}$.

(4) $\overline{\mathbf{r k}}_{\mathcal{C}}^{\prime}(A)$ is the smallest $n$ (possibly $\infty$ ) such that products of at most $n$ elements of the class $\mathcal{C}$ are dense in $\bar{P}_{\mathcal{C}}^{\prime}(A)$.

(5) $\quad N_{\mathcal{C}}^{\prime}(n, d)=\sup _{\operatorname{dim}(X) \leq d} \operatorname{rk}_{\mathcal{C}}^{\prime}\left(C(X) \otimes M_{n}\right)$, where $X$ runs through all compact metric spaces of dimension at most $d$.

We further set $\operatorname{cel}^{\prime}(A)=\sup \left\{\operatorname{cel}(u): u \in U_{0}(A)^{\prime}\right\}$, and define

$$
N^{\prime}(n, d)=\sup _{\operatorname{dim}(X) \leq d} \operatorname{cel}^{\prime}\left(C(X) \otimes M_{n}\right)
$$

using the same spaces $X$ as before. 
Note that $\mathrm{rk}_{\mathcal{C}}^{\prime}(C(X))=\overline{\mathrm{rk}}_{\mathcal{C}}^{\prime}(C(X))=1$ for $\mathcal{C}$ equal to any of $\mathcal{A}, \mathcal{S}$, and $\mathcal{U}$. We have thus recovered the behavior of Proposition 1.4(2).

4.8 Remark. For $A=C(X) \otimes M_{n}$, Theorems 2.10(1) and 3.10(1) imply that $\operatorname{inv}_{0}(A)^{\prime} \cap U_{0}(A)=U_{0}(A)^{\prime}$. So we could have used $\operatorname{inv}_{0}(A)^{\prime}$ in the definition of $\operatorname{rk}_{\mathcal{U}}^{\prime}(A)$ too.

We also have $\operatorname{inv}_{0}(A)^{\prime} \cap U_{0}(A)=U_{0}(A)^{\prime}$ for the algebras considered in [4] and [26]. In general, however, it seems to be unknown when this relation holds.

4.9 Remark. We can also analogously define $P_{\mathcal{C}}^{\prime}(A), \operatorname{rk}_{\mathcal{C}}^{\prime}(A)$, etc. for other classes mentioned in or before Definition 1.1. The analogs of the theorems in Section 2 and 3 still hold, with the trivial modification that one must impose the condition $\operatorname{det}(a)=1$ in the characterizations of $P_{\mathcal{C}}^{\prime}(A)$ and $\bar{P}_{\mathcal{C}}{ }^{\prime}(A)$. (Note that the elements used to prove the lower bounds in all of these theorems actually have determinant 1.)

We now present the main theorems, starting with the one for exponential length.

4.10 Theorem. (1) $\quad$ cel $(u)<\infty$ for all $u \in U_{0}\left(C(X) \otimes M_{n}\right)^{\prime}$.

(2) $N^{\prime}(n, d)<\infty$ for $n \geq 1$ and $0 \leq d<\infty$.

(3) For fixed $d<\infty$, we have $\lim _{n \rightarrow \infty} \sup N^{\prime}(n, d) \leq 4 \pi$.

(4) If $X$ is a compact manifold with boundary and $n \geq 2$, then

$$
\operatorname{cel}^{\prime}\left(C(X) \otimes M_{n}\right)>\pi\left(\frac{\operatorname{dim}(X)-2}{n^{2}-1}-2\right) .
$$

Proof. (1) Actually, $\operatorname{cel}(u)<\infty$ for any $u \in U_{0}(A)$ for any unital $\mathrm{C}^{*}$-algebra $A$. See [24].

(2) This is just Lemma 3.3.

(3) The proof of Theorem 3.3 of [13] gives, for each positive integer $d$ and $\varepsilon>0$, a number $M(d, \varepsilon)$ such that whenever $\operatorname{dim}(X) \leq d$ and $u$ is a homotopically trivial element of $C\left(X, S U_{n}\right)$ with $n \geq M(d, \varepsilon)$, then there exist selfadjoint $h_{1}, h_{2}, h_{3} \in C(X) \otimes M_{n}$ such that $\left\|u-\exp \left(i h_{1}\right) \exp \left(i h_{2}\right) \exp \left(i h_{3}\right)\right\|<$ $\varepsilon$. (Note that the first step in this proof, which applies to an arbitrary $u \in U_{0}\left(C(X) \otimes M_{n}\right)$, is to reduce to the case $\operatorname{det}(u)=1$.) An examination of the proof shows that the $h_{1}$ chosen in it satisfies $\left\|h_{1}\right\| \leq 2 \pi$. Similarly, going back to [12] in Step 7 of this proof, we get $\left\|h_{2}\right\|,\left\|h_{3}\right\| \leq \pi$. Therefore $\operatorname{cel}(u) \leq 4 \pi+2 \arcsin (\varepsilon / 2)$, using Proposition 2.4 of [24]. So $\operatorname{cel}^{\prime}\left(C(X) \otimes M_{n}\right) \leq 4 \pi+2 \arcsin (\varepsilon / 2)$ for all large enough $n$.

(4) Theorem 2.6 of [24] shows that if $\operatorname{cel}(u) \leq l \pi$, then $u$ is a limit of products of $l$ exponentials. Now use Proposition 3.2. 
4.11 Remark. The paths whose lengths are estimated in parts (2) and (3) of this theorem are not required to have determinant 1 except at the endpoints. It might seem more appropriate to require them to lie entirely in $U_{0}(A)^{\prime}$. With this extra condition, (2) still holds, and still follows from Lemma 3.3. One can show that (3) holds with $4 \pi$ replaced by $6 \pi$. We omit the details, but note that it is fairly easy to get the estimate $10 \pi$. One replaces each $h_{j}$ by $h_{j}=-\frac{1}{n} \operatorname{tr}\left(h_{j}\right) \cdot 1$, which at most doubles the norm. One does the same thing with $h_{4}=-i \log \left(\exp \left(-i h_{3}\right) \exp \left(-i h_{2}\right) \exp \left(-i h_{1}\right) u\right)$. From this, one gets a path of length not much more than $8 \pi$ to a locally constant scalar element of $C\left(X, S U_{n}\right)$. Now observe that a scalar in $S U_{n}$ can be connected to 1 by a path of length less than $2 \pi$.

4.12 Theorem. (1) $P_{\mathcal{A}}^{\prime}\left(C(X) \otimes M_{n}\right)=\bar{P}_{\mathcal{A}}^{\prime}\left(C(X) \otimes M_{n}\right)=\left\{a \in \operatorname{inv}_{0}(C(X) \otimes\right.$ $\left.\left.M_{n}\right): \operatorname{det}(a)=1\right\}$.

(2) $N_{\mathcal{A}}^{\prime}(n, d)<\infty$ for $n \geq 1$ and $0 \leq d<\infty$.

(3) For fixed $d<\infty$ we have $\lim _{n \rightarrow \infty} \sup N_{\mathcal{A}}^{\prime}(n, d) \leq 10$.

(4) If $X$ is a compact manifold with boundary and $n \geq 2$, then

$$
\overline{\mathrm{rk}}_{\mathcal{A}}^{\prime}\left(C(X) \otimes M_{n}\right) \geq \frac{\operatorname{dim}(X)-2}{n^{2}-1}-1 \text {. }
$$

4.13 Theorem. (1) $P_{\mathcal{S}}^{\prime}\left(C(X) \otimes M_{n}\right)=\bar{P}_{\mathcal{S}}^{\prime}\left(C(X) \otimes M_{n}\right)=\left\{a \in \operatorname{inv}_{0}(C(X) \otimes\right.$ $\left.\left.M_{n}\right): \operatorname{det}(a)=1\right\}$.

(2) $N_{\mathcal{S}}^{\prime}(n, d)<\infty$ for $n \geq 1$ and $0 \leq d<\infty$.

(3). For fixed $d<\infty$ we have $\lim _{n \rightarrow \infty} \sup N_{\mathcal{S}}^{\prime}(n, d) \leq 10$.

(4) If $X$ is a compact manifold with boundary and $n \geq 2$, then

$$
\overline{\mathrm{rk}}_{\mathcal{S}}^{\prime}\left(C(X) \otimes M_{n}\right) \geq \frac{\operatorname{dim}(X)-2}{2\left(n^{2}-1\right)} .
$$

4.14 Theorem. (1) $P_{\mathcal{U}}^{\prime}\left(C(X) \otimes M_{n}\right)=\bar{P}_{\mathcal{U}}^{\prime}\left(C(X) \otimes M_{n}\right)=\left\{a \in U_{0}(C(X) \otimes\right.$ $\left.\left.M_{n}\right): \operatorname{det}(u)=1\right\}$.

(2) $N_{\mathcal{U}}^{\prime}(n, d)<\infty$ for $n \geq 1$ and $0 \leq d<\infty$.

(3) For fixed $d<\infty$ we have $\lim _{n \rightarrow \infty} \sup N_{\mathcal{U}}^{\prime}(n, d) \leq 9$.

(4) If $X$ is a compact manifold with boundary and $n \geq 2$, then

$$
\overline{\mathrm{rk}}_{\mathcal{U}}^{\prime}\left(C(X) \otimes M_{n}\right) \geq 2 \frac{\operatorname{dim}(X)-2}{n^{2}-1}-3 .
$$

Proof of Theorems 4.12. 4.13. and 4.14. (1) This is immediate from Proposition 4.5 and the remark before Definition 4.7 in all three cases. 
(2), (3) The proof of part (3) of Proposition 4.5 shows, in exactly the same way, that the same inequalities hold for $\mathrm{rk}_{\mathcal{C}}^{\prime}$ in place of $\mathrm{rk}_{\mathcal{C}}$ and cel in place of cel. Part (2) now follows in all three cases from the corresponding part of Theorem 4.10. For (3), use Theorem 4.10(3) to choose $N$ such that $n \geq N$ and $\operatorname{dim}(X) \leq d$ imply $\operatorname{cel}^{\prime}\left(C(X) \otimes M_{n}\right)<9 \pi / 2$. For such $n$ and $X$, we have $\mathrm{rk}_{\mathcal{A}}^{\prime}\left(C(X) \otimes M_{n}\right), \mathrm{rk}_{\mathcal{S}}^{\prime}\left(C(X) \otimes M_{n}\right)<11$ and $\mathrm{rk}_{\mathcal{U}}^{\prime}\left(C(X) \otimes M_{n}\right)<10$.

(4) In Theorem 4.12, we note that if $a, b \in C(X) \otimes M_{n}$ are accretive, then Theorem 2.22 of [29] implies that $\operatorname{sp}(a(x) b(x))$ contains no nonpositive real numbers. Therefore products of two accretive elements are exponentials. Now use Proposition 2.2. In Theorem 4.13, we simply observe that elements with spectrum in the right half plane have logarithms, and apply Proposition 2.2. In Theorem 4.14, we note that the product of two accretive unitaries has exponential length less than $\pi$ by Lemma 4.4(4), and so is the exponential of a skewadjoint element. Use Proposition 3.2.

\section{References}

[1] R. Bhatia, Perturbation Bounds for Matrix Eigenvalues, Pitman Research Notes in Math. no. 162, Longman Scientific and Technical, Harlow, Britain, 1987.

[2] B. Blackadar, K-Theory for Operator Algebras, MSRI publications no. 5, SpringerVerlag, New York, Berlin, Heidelberg, London, Paris, Tokyo, 1986.

[3] A. Calder and J. Siegel, On the width of homotopies, Topology, 19 (1980), 209-220.

[4] P. de la Harpe and G. Skandalis, Produits finis de commutateurs dans les $\mathrm{C}^{*}$. algèbres, Ann. Inst. Fourier (Grenoble), 34, no. 4 (1984), 169-202.

[5] R. K. Dennis and L. N. Vaserstein, On a question of M. Newman on the number of commutators, J. Algebra, 118 (1988), 150-161.

[6] R. Engelking, Dimension Theory, North-Holland, Amsterdam, Oxford, New York, 1978.

[7] P. A. Fillmore, Notes on Operator Theory, Van Nostrand Reinhold, New York, Cincinnati, Toronto, London, Melbourne, 1970.

[8] P. R. Halmos, A Hilbert Space Problem Book, Van Nostrand, New York, Toronto, London, Melbourne, 1967.

[9] T. Kato, Perturbation Theory for Linear Operators (2nd corrected printing of the 2nd ed.), Springer-Verlag, Berlin, Heidelberg, New York, Tokyo, 1984.

[10] M. Khalkhali, C. Laurie, B. Mathes, and H. Radjavi, Approximation by products of positive operators, J. Operator Theory, 29 (1993), 237-247.

[11] N. C. Phillips, Simple $\mathrm{C}^{*}$-algebras with the property weak $(F U)$, Math. Scand., 69 (1991), 127-151.

[12] _ Approximation by unitaries with finite spectrum in purely infinite $\mathrm{C}^{*}$ algebras, J. Functional Analysis, 120 (1994), 98-106.

[13] - How many exponentials?, Amer. J. Math., 116 (1994), 1513-1543.

[14] The rectifiable metric on the space of projections in a $\mathrm{C}^{*}$-algebra, International J. Math., 3 (1992), 679-698. 
[15] - The $\mathrm{C}^{*}$ projective length of n-homogeneous $\mathrm{C}^{*}$-algebras, J. Operator Theory, 31 (1994), 253-276.

[16] — A survey of exponential rank, $\mathrm{C}^{*}$-Algebras: 1943-1993, A Fifty Year Celebration, Contemporary Math., Vol. 167, Amer. Math. Soc., Providence RI, 1994, 353-399.

[17] _ Every invertible Hilbert space operator is a product of seven positive operators, Canad. Math. Bulletin, 38 (1995), 230-236.

[18] N. C. Phillips and J. R. Ringrose, Exponential rank in operator algebras, Current Topics in Operator Algebras, H. Araki etc. (Eds.), World Scientific, Singapore, New Jersey, London, Hong Kong, 1991, 395-413.

[19] T. Quinn, Factorization in $\mathrm{C}^{*}$-Algebras: Products of Positive Operators, Ph.D. Thesis, Dalhousie University, Halifax, 1992.

[20] , Products of decomposable positive operators, preprint.

[21] H. Radjavi, Products of Hermitian matrices and symmetries, Proc. Amer. Math. Soc., 21 (1969), 369-372.

[22] M. A. Rieffel, Dimension and stable rank in the $K$-theory of $\mathrm{C}^{*}$-algebras, Proc. London Math. Soc., (3) 46 (1983), 301-333.

[23] - The homotopy groups of the unitary groups of noncommutative tori, J. Operator Theory, 17 (1987), 237-254.

[24] J. R. Ringrose, Exponential length and exponential rank in $\mathrm{C}^{*}$-algebras, Proc. Royal Soc. Edinburgh, Ser. A., 121 (1992), 55-71.

[25] A. R. Sourour, A factorization theorem for matrices, Linear Multilinear Alg., 19 (1986), 141-147.

[26] K. Thomsen, Finite sums and products of commutators in inductive limit $\mathrm{C}^{*}$ algebras, Ann. Inst. Fourier (Grenoble), 43, no.1 (1993), 225-249.

[27] L. N. Vaserstein, Stable rank of rings and dimensionality of topological spaces, Funktsional. Anal. i Prilozhen, 5 no. 2 (1971), 17-27 (in Russian); English translation in Functional Anal. Appl., 5 (1971), 102-110.

[28] , Reduction of a matrix depending on parameters to a diagonal form by addition operation, Proc. Amer. Math. Soc., 103 (1988), 741-746.

[29] P. Y. Wu, The operatorfactorization problems, Linear Alg. Appl., 117 (1989), 35-63.

[30] _ Products of normal operators, Can. J. Math., 40 (1988), 1322-1330.

Received September 14, 1993 and revised April 5, 1995. Research partially supported by NSF grant DMS 91-06285.

\section{UNIVERSITY OF OREgON}

EUGENE, OR 97403-1222

E-mail address: phillips@math.uoregon.edu 



\section{PACIFIC JOURNAL OF MATHEMATICS}

Volume $174 \quad$ No. $1 \quad$ May 1996

A distance formula for algebras on the disk

CHRISTOPHER J. BISHOP

Rigidity of isotropic maps

FERNANDO CUKIERMAN

The Schwartz space of a general semisimple Lie group. V. Schwartz

class wave packets

REBECCA A. HERB

Rational polynomials with a $\mathbf{C}^{*}$-fiber

SHULIM KALIMAN

Linear combinations of logarithmic derivatives of entire functions with 195 applications to differential equations

Joseph B. Miles and JoHn Rossi

Factorization problems in the invertible group of a homogeneous $C^{*}$-algebra

N. Christopher PHiLlips

Higher order estimates in complex interpolation theory

RICHARD ROCHBERG

Braid commutators and Vassiliev invariants

TED STANFORD

On the Cauchy problem for a singular parabolic equation 